\title{
DISSECTING A RESONANCE WEDGE ON HETEROCLINIC BIFURCATIONS
}

\author{
ALEXANDRE A. P. RODRIGUES \\ CENTRO DE MATEMÁTICA DA UNIV. DO PORTO \\ RUA DO CAMPO ALEGRE, 687, 4169-007 PORTO, PORTUGAL
}

\begin{abstract}
This article studies routes to chaos occurring within a resonance wedge for a 3-parametric family of differential equations acting on a 3-sphere. Our starting point is an autonomous vector field whose flow exhibits a weakly attracting heteroclinic network made by two 1-dimensional connections and a 2-dimensional separatrix between two equilibria with different Morse indices. After changing the parameters, while keeping the 1-dimensional connections unaltered, we concentrate our study in the case where the 2-dimensional invariant manifolds of the equilibria do not intersect.

We derive the first return map near the network and we reduce the analysis of the system to a 2-dimensional map on the cylinder. Complex dynamical features arise from a discretetime Bogdanov-Takens singularity, which may be seen as the organizing center by which one can obtain infinitely many attracting tori, strange attractors, infinitely many sinks and nontrivial contracting wandering domains. These dynamical phenomena occur within a structure that we call resonance wedge. As an application, we may see the "classical" Arnold tongue as a projection of a resonance wedge. The results are general, extend to other contexts and lead to a fine-tuning of the theory.
\end{abstract}

\section{INTRODUCTION}

To date there has been very little systematic investigation of the effects of perturbations that break an invariant torus, despite being natural for the modelling of many biological and physical effects [2, 32, 33, 40, 49, 54]. In this paper, we describe the transition from regular dynamics to chaos associated to the Torus-breakdown Theory partially described in Afraimovich and Shilnikov [1, applied to a specific heteroclinic configuration involving 2dimensional connecting manifolds (continuum of connections in the terminology of [1]]).

In studying bifurcations associated to Torus-breakdown, it is natural to examine the bifurcation diagram in terms of Arnold tongues [8]. In planar maps, a $\frac{p}{q}$-resonance tongue can be defined as the locus, in the parameter space, where periodic points with rotation number $\frac{p}{q}$ exist, for $p, q \in \mathbb{N}$; in the engineering literature, these solutions are also called $p: q$ phaselocked [22, 27. The way in which resonance tongues overlap and evolve indicates how the rotational dynamics is changing.

Although tongues corresponding to different rotation numbers can overlap, in general there is no path where a periodic orbit from a resonance tongue becomes a periodic orbit from

Date: June 3, 2021.

2010 Mathematics Subject Classification. 34C28; 34C37; 37D05; 37D45; 37G35

Keywords: Heteroclinic bifurcations; Torus-breakdown; Resonance wedge; Arnold tongue; Strange attractors.

AR was partially supported by CMUP (UID/MAT/00144/2019), which is funded by FCT with national (MCTES) and European structural funds through the programs FEDER, under the partnership agreement PT2020. AR also acknowledges financial support from Program INVESTIGADOR FCT (IF/00107/2015). 
another tongue [15, 22. A singular phenomenon has been described by Kirk [32, who studied resonance zones for a 3-dimensional system that corresponds to the normal form associated to a codimension-two Hopf-zero singularity. Such a normal form is perturbed with nonsymmetric terms breaking the axial symmetry and the phenomenon of "merging of resonance wedges" has been described: far from the torus bifurcation, a periodic orbit modifies its shape and collapses with another periodic orbit coming from another tongue.

Assuming a strong 1-dimensional contracting direction, the structure of the Arnold tongue has been given by Arnold [8], Boyland [15] and Herman [27] who reduced the study of Torusbreakdown effects to the "canonical family" on the circle:

$$
x \mapsto \quad \mapsto+a+\frac{b}{2 \pi} \sin (2 \pi x) \quad \bmod 1, \quad a, b \in \mathbb{R} .
$$

When the radial contracting foliation on the torus is lost, the bifurcation structure of the circle maps family is inadequate to explain the diverse phenomena that accompany the loss of the attracting torus and it is here that the study of resonance wedges plays an important role. Resonance tongues are associated to a wide range of behaviours such us: the existence of quasiperiodic solutions, sinks, saddle-node bifurcations, homoclinic orbits, bistability, rotational horseshoes and strange attractors (either "large" or "small" according to Broer et al [17]). Early papers in this context are [1, 2, 17, 10]. We address the reader to [3, 9, 15, 17, 25, 47] for more information on the subject. For a tutorial, see Shilnikov et al [55]. New directions of the theory and applications to periodiocally-kicked differential equations can be found in [13, 14, 20, 52, 57].

The goal of this paper is to construct a 3-parameter bifurcation diagram for a concrete configuration associated to a weakly attracting heteroclinic network with two saddle-foci previously studied in [38, 52], the Bykov attractor. Our study has been motivated by numerical results obtained in $[6,20,21$.

Our purpose in writing this paper is not only to point out the range of phenomena that can occur when an invariant torus is broken, but to bring to the foreground the techniques that have allowed us to reach these conclusions in a relatively straightforward manner. These mechanisms are not limited to the heteroclinic network considered here.

1.1. The novelty. While some progress has been made, both numerically and analytically, the number of explicit configurations whose flows have an invariant torus and for which the Torus-Breakdown description is available, has remained small.

By studying unfoldings of a Bykov attractor, we are able to delineate the ways in which the first return map to a cross section can make the transition from a single rotation number to an interval of rotation number 1 . As a continuation of the project started in [52, we will prove analytically that a sequence of discrete-time Bogdanov-Takens bifurcations organise the dynamics that appear in the unfolding. Besides, within the Arnold wedge, we numerically find surfaces corresponding to the following bifurcations: Hopf, period-doubling and the transition node $\mapsto$ focus. Our results agree well with the literature about heteroclinic bifurcations, Arnold tongues and the scenarios described in [9, 10, 47.

Our object of study is not "just another dynamical system", but representative for the case of 3-dimensional dissipative flows admitting 2-dimensional connections that are pulled apart. In similar models, the corresponding phenomenology should contain no further secrets.

\footnotetext{
${ }^{1}$ The existence of rotational horseshoes, described in Appendix A.8, is responsible for the existence of an interval of rotation number - see 45 .
} 
1.2. Physical setting. Our study allows us to understand the bifurcations from an invariant torus to strange attractors that appear in Ruelle and Takens [53] and Langford [40] (see also $\S 6.2$ of [20]). In the context of turbulent flows, the author of [40] studied a two-parameter unfolding a Hopf-zero singularity and proved that axisymmetric perturbations generate an invariant torus. By slightly breaking the symmetry, Langford prove that the flow becomes more and more turbulent with fractal basins of attraction as a consequence of the emergence strange attractors. The bifurcations described in our paper have similarities with those described in [54] in the context of a low-order atmospheric circulation model.

1.3. The structure. In Section 2, we describe precisely our object of study and we review the literature related to it. In Section 3 we state the main results of the article. The coordinates and other notation used in the rest of the article are presented in Section 4 to prove the main results of the manuscript in Section 5 .

We refine the structure of Arnold tongues which appear in the context of heteroclinic bifurcations on Section 6. In Section 7, we briefly illustrate our theoretical results with an example explored in [21]. Symmetry-breaking effects will be described.

We finish the article with a discussion in Section 8 about the consequences of our findings. Dynamics similar to what we described is expected to occur near periodically forced attracting heteroclinic cycles. For reader's convenience, we have compiled at the end of the article (Appendix A) a list of basic definitions.

\section{SEtTing AND StATE OF ART}

In this section, we describe the main hypotheses about the weakly heteroclinic network we are considering. We postpone the technical definitions used in this section to Appendix $\mathrm{A}$.

2.1. Starting point. For $\varepsilon>0$ small, consider the 3 -parameter family of $C^{3}$-smooth differential equations

$$
\dot{x}=f_{(A, \lambda, \omega)}(x) \quad x \in \mathbb{S}^{3} \subset \mathbb{R}^{4} \quad A, \lambda \in[0, \varepsilon], \quad \omega \in \mathbb{R}^{+}
$$

where $\mathbb{S}^{3}$ represents the unit three-sphere, endowed with the usual topology. Let us denote by $\varphi_{(A, \lambda, \omega)}(t, x), t \in \mathbb{R}$, the flow associated to (2.1) satisfying the following properties for $A=\lambda=0$ and $\omega \in \mathbb{R}^{+}$:

(P1) There are two different equilibria, say $O_{1}$ and $O_{2}$.

(P2) The eigenvalues of $D f_{X}$ are:

(P2a) $E_{1}$ and $-C_{1} \pm \omega i$ where $C_{1}, E_{1}>0$, for $X=O_{1}$;

(P2b) $-C_{2}$ and $E_{2} \pm \omega i$ where $C_{2}, E_{2}>0$, for $X=O_{2}$.

For $W \subseteq \mathbb{S}^{3}$, denoting by $\bar{W}$ the closure of $W$, we also assume that:

(P3) The manifolds $\overline{W^{u}\left(O_{2}\right)}$ and $\overline{W^{s}\left(O_{1}\right)}$ coincide and the set $\overline{W^{u}\left(O_{2}\right) \cap W^{s}\left(O_{1}\right)}$ consists of a 2-dimensional sphere $\left(W^{u}\left(O_{2}\right) \cap W^{s}\left(O_{1}\right)\right.$ is called the $2 D$-connection).

and 
(P4) There are two trajectories, say $\gamma_{1}, \gamma_{2}$, contained in $W^{u}\left(O_{1}\right) \cap W^{s}\left(O_{2}\right)$, each one in each connected component of $\mathbb{S}^{3} \backslash \overline{W^{u}\left(O_{2}\right)}\left(\gamma_{1}, \gamma_{2}\right.$ are called the $1 D$-connections).

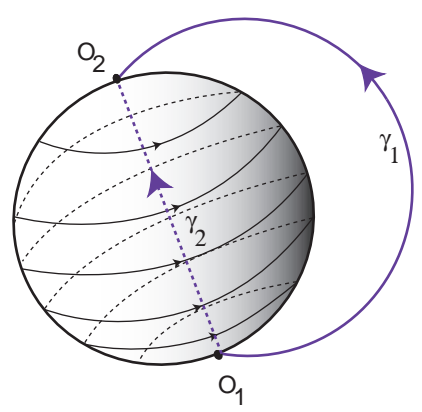

Figure 1. Scheme of the attractor $\Gamma$ satisfying $(\mathbf{P} 1)-(\mathbf{P} 4)$, for $A=\lambda=0$ and $\omega>0$.

The equilibria $O_{1}$ and $O_{2}$, the $2 D$-connection referred in (P3) and the two trajectories $\gamma_{1}, \gamma_{2}$ of (P4) build a heteroclinic network, that we denote by $\Gamma$. This network, illustrated in Figure 1, has two cycles. Assuming that the set of eigenvalues of $D f_{\mathrm{O}_{1}}$ and $D f_{\mathrm{O}_{2}}$ satisfy

$$
\text { (P5) } \frac{C_{1} C_{2}}{E_{1} E_{2}} \gtrsim 1
$$

the network $\Gamma$ is asymptotically stable (proof in [38]): with exception of the origin, all trajectories are forward attracted to $\Gamma$. As a consequence, we may find a neighborhood $\mathcal{U}$ of $\Gamma$ having its boundary transverse to the flow and such that every solution starting in $\mathcal{U}$ is asymptotic to $\Gamma$. The set $\Gamma$ is usually called Bykov attractor ${ }^{2}$.

2.2. Chirality: a topological assumption. There are two possibilities for the geometry of the flow around the saddle-foci of $\Gamma$, depending on the direction the trajectories turn around the $1 D$-connections. This is related to the topological concept of chirality introduced in [37].

Let $V_{1}$ and $V_{2}$ be small disjoint neighborhoods of $O_{1}$ and $O_{2}$ with boundaries $\partial V_{1}$ and $\partial V_{2}$, respectively. These neighborhoods will be precisely constructed in Section 4 . Trajectories starting at $\partial V_{1} \backslash W^{s}\left(O_{1}\right)$ near $W^{s}\left(O_{1}\right)$ go into the interior of $V_{1}$ in positive time, then follow one of the solutions in $\left[O_{1} \rightarrow O_{2}\right]$, go inside $V_{2}$, come out at $\partial V_{2}$ and then return to $\partial V_{1}$ (see Figure 2). This trajectory is not closed since $\Gamma$ is attracting.

Let $\mathcal{Q}$ be a piece of trajectory like this from $\partial V_{1}$ to $\partial V_{1}$. Within $\partial V_{1} \backslash W^{s}\left(O_{1}\right)$, join its starting point to its end point by a segment as in Figure 2, forming a closed curve, which we call the loop of $\mathcal{Q}$. By construction, the loop of $\mathcal{Q}$ and $\Gamma$ are disjoint closed sets.

Definition 1. 37] We say that $O_{1}$ and $O_{2}$ in $\Gamma$ have the same chirality if the loop of every trajectory starting near $O_{1}$ is linked to $\Gamma$ (i.e the trajectories cannot be disconnected by an isotopy). Otherwise, we say that $O_{1}$ and $O_{2}$ have different chirality.

The next hypothesis may be written as:

(P6) The saddle-foci $O_{1}$ and $O_{2}$ have the same chirality. 


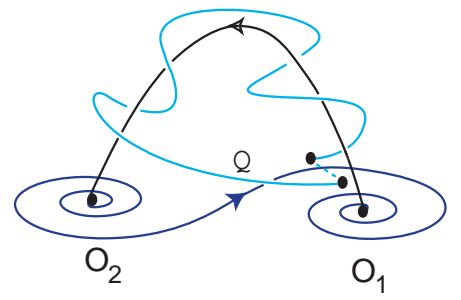

Figure 2. Illustration of Property (P6): the saddle-foci $O_{1}$ and $O_{2}$ have the same chirality.

For $r \geq 3$, denote by $\mathfrak{X}^{r}\left(\mathbb{S}^{3}\right)$, the set of 3-parameter family of $C^{3}$-vector fields on $\mathbb{S}^{3}$ satisfying Properties (P1) $-(\mathbf{P} 6)$, endowed with the $C^{r}$-topology.

2.3. Perturbing terms. Concerning the effect of $A, \lambda$ and $\omega$ on the dynamics of (2.1), we assume that:

(P7) For $A>\lambda \geq 0$ and $\omega \in \mathbb{R}^{+}$, the two trajectories within $W^{u}\left(O_{1}\right) \cap W^{s}\left(O_{2}\right)$ persist.

By the Kupka-Smale Theorem, generically the invariant 2-dimensional manifolds $W^{u}\left(O_{2}\right)$ and $W^{s}\left(O_{1}\right)$ are transverse (either intersecting or not):

(P8a) For $A, \lambda \geq 0$ and $\omega \in \mathbb{R}^{+}$, the manifolds $W^{u}\left(O_{2}\right)$ and $W^{s}\left(O_{1}\right)$ intersect transversely.

(P8b) For $A, \lambda \geq 0$ and $\omega \in \mathbb{R}^{+}$, the manifolds $W^{u}\left(O_{2}\right)$ and $W^{s}\left(O_{1}\right)$ do not intersect.

and

(P9) Up to high order terms in $x, y$, the transitions along the connections $\left[O_{1} \rightarrow O_{2}\right]$ and $\left[\mathrm{O}_{2} \rightarrow \mathrm{O}_{1}\right]$ are given, in the local coordinates that will be defined in Section 4 , by the Identity map and by

$$
(x, y) \mapsto(x, y+A+\lambda \Phi(x))
$$

respectively, where $\Phi: \mathbb{S}^{1} \rightarrow \mathbb{S}^{1}$ is a Morse function with at least two non-degenerate critical points $\left(\mathbb{S}^{1}=\mathbb{R}(\bmod 2 \pi)\right)$. This assumption will be clearer later.

(a)

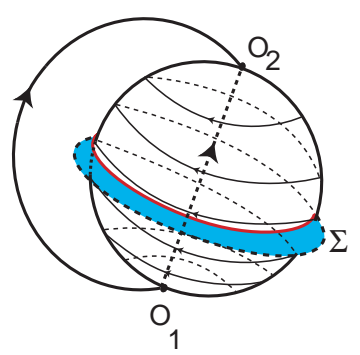

(b)

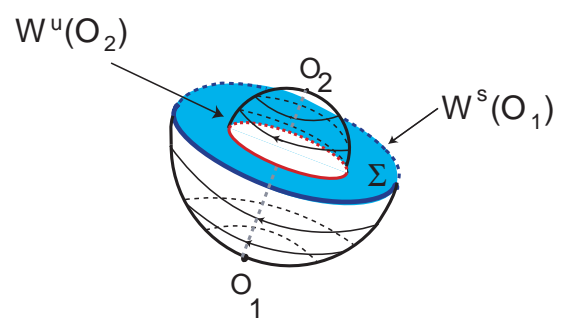

(c)

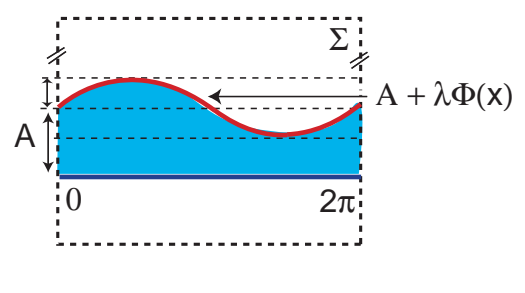

Figure 3. Illustration of Properties (P8b) and (P9), where $\Sigma$ represents a global cross section to $\Gamma$. Double bars mean that the sides are identified.

\footnotetext{
${ }^{2}$ The terminology Bykov is a homage to V. Bykov who dedicated his latest research projects to similar cycles [18, 19].
} 
2.4. Constants. Once for all, we define the following notation that will be used throughout the present manuscript:

$$
\begin{array}{ccc}
\delta_{1}=\frac{C_{1}}{E_{1}}>0 & \delta_{2}=\frac{C_{2}}{E_{2}}>0 & \delta=\delta_{1} \delta_{2} \gtrsim 1 \\
\omega_{\ell}^{\star}=\frac{2 \ell \pi(\delta-1)}{\ln \delta}>0 & K=\frac{E_{2}+C_{1}}{E_{1} E_{2}}>0 & \\
M=\delta^{\frac{1}{1-\delta}}-\delta^{\frac{\delta}{1-\delta}} & \mu=(A, \lambda, \omega) & \nu=\left(A, \lambda_{0}, \omega\right), \lambda_{0}>0 \text { fixed }
\end{array}
$$

2.5. Digestive remarks about the hypotheses. We discuss the Hypotheses (P1)-(P9), stressing that they are natural in several settings. An illustrative scheme of the Hypotheses has been summarized in Table 1 of [52].

Remark 2.1. Although the fully description of the bifurcations associated to the heteroclinic attractor $\Gamma$ is a phenomenon of codimension three [35, 36], the setting described by (P1)(P9) is natural in symmetric contexts [4, 38, and also in some unfoldings of the Hopf-zero singularity [12, 23, 54].

Remark 2.2. Hypothesis (P7) corresponds to the partial symmetry-breaking considered in Section 2.4 of [36]. The setting described by $(\mathbf{P 1})-(\mathbf{P} 8 \mathbf{b})$ and $(\mathbf{P 9})$ generalizes Case (4) of $[52]$.

Remark 2.3. The first hit of $W^{u}\left(O_{2}\right)$ and $W^{s}\left(O_{1}\right)$ to a global cross section $\Sigma$ are two closed curves (see Figure 3); the distance between these two curves can be written as

$$
A+\lambda \Phi(x), \quad x \in \mathbb{S}^{1},
$$

which may be seen as an approximation of the Melnikov integral associated to the intersection of $W^{u}\left(O_{2}\right)$ and $W^{s}\left(O_{1}\right)$ [26, $\left.\S 4.5\right]$.

Remark 2.4. Variable $\omega$ represents the speed of rotation of the saddle-foci. Using different imaginary parts on the complex eigenvalues of $D f_{\mathrm{O}_{1}}$ and $D f_{\mathrm{O}_{2}}$ would complicate the expression of $K \omega$, without any qualitative benefits in the final result.

Remark 2.5. Derivations using a more general form for the transition $\left[O_{1} \rightarrow O_{2}\right]$ have been performed in Section 6 of [50]. The transition along $\left[O_{2} \rightarrow O_{1}\right]$ corresponds to the expected unfolding from the coincidence of the 2-dimensional invariant manifolds at $f_{(0,0, \omega)}, \omega \in \mathbb{R}^{+}$.

Remark 2.6. The technical Hypothesis (P9) modulates the two generic possibilities given by (P8a) and (P8b):

$$
\begin{aligned}
& \lambda>A \geq 0, \omega \in \mathbb{R}^{+} \quad \Leftrightarrow \quad(\mathbf{P} 8 \mathbf{a}): W^{u}\left(O_{2}\right) \text { and } W^{s}\left(O_{1}\right) \text { intersect transversely; } \\
& A>\lambda \geq 0, \omega \in \mathbb{R}^{+} \quad \Leftrightarrow \quad(\mathbf{P} 8 \mathbf{b}): W^{u}\left(O_{2}\right) \text { and } W^{s}\left(O_{1}\right) \text { do not intersect. }
\end{aligned}
$$


Assumption (P9) governs the transition maps along the heteroclinic connections and is necessary to make precise computations. All results are valid for any $2 \pi$-periodic non-constant Morse function defined on $[-1,1]$.

2.6. State of art. In this section, we give an overview of results for the class of vector fields satisfying either (P1)-(P8a)-(P9) or (P1) $-(\mathbf{P} 8 b)-(\mathbf{P 9})$.

2.6.1. Heteroclinic tangle: If $f_{(A, \lambda, \omega)} \in \mathfrak{X}^{3}\left(\mathbb{S}^{3}\right)$ and satisfies $(\mathbf{P} 7)-(\mathbf{P} 8 \mathbf{a})-(\mathbf{P 9})$, then the 2dimensional invariant manifolds $W^{u}\left(O_{2}\right)$ and $W^{s}\left(O_{1}\right)$ meet transversely, giving rise to a union of Bykov cycles [19, 38. The dynamics in the maximal invariant set inside $\mathcal{U}$, contains the suspension of horseshoes accumulating on the network described in [5, 35, 36, 50. Near the original heteroclinic attractor $\Gamma$, the flow contains infinitely many homoclinic tangencies and sinks with long periods, coexisting with sets with positive entropy, giving rise the so called quasi-stochastic attractors [24]; more details in Appendices A.2 and A.3.

2.6.2. Torus-breakdown. If $f_{(A, \lambda, \omega)} \in \mathfrak{X}^{3}\left(\mathbb{S}^{3}\right)$ and satisfies $(\mathbf{P} 7)-(\mathbf{P} 8 \mathbf{b})-(\mathbf{P 9})$, then the 2dimensional invariant manifolds of the saddle-foci do not intersect. According to [52, in the bifurcation diagram $(\omega, \lambda / A)$ of Figure 4 , there are two curves, the graphs of $h_{1}$ and $h_{2}$, such that, for all $\omega \in \mathbb{R}^{+}$, we have $h_{1}(\omega)<h_{2}(\omega)$ and:

(1) Attracting torus: the region below the graph of $h_{1}$ corresponds to parameters whose flow has an attracting normally hyperbolic 2-torus with zero topological entropy Theorem B of [52]. In the bifurcation parameter $(\omega, \lambda / A)$, there exists a set of positive Lebesgue measure for which the whole torus is the minimal attractor.

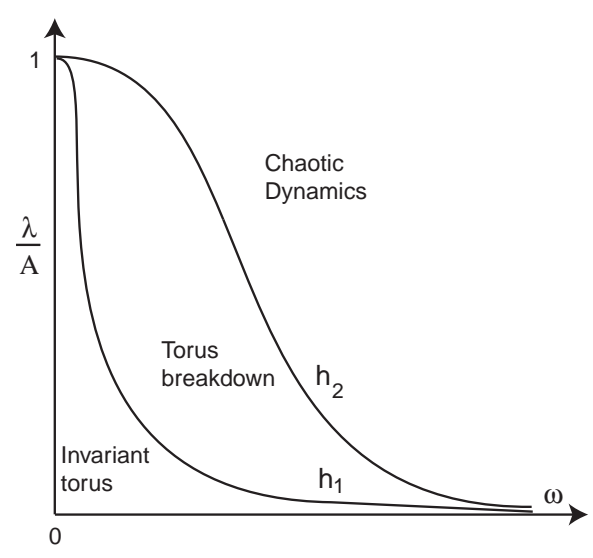

FiguRE 4. Summary of the results of [52]: location of the attracting 2-torus and chaotic regions with respect to $\omega$ and $\frac{\lambda}{A}$, for a vector field $f_{(A, \lambda, \omega)} \in \mathfrak{X}_{\mathrm{Byk}}^{3}\left(\mathbb{S}^{3}\right)$.

(2) Transitional dynamics: for a fixed $\omega>0$, in the transition from $h_{1}(\omega)$ to $h_{2}(\omega)$, the attracting torus breaks. It starts to disintegrate into a finite collection of periodic saddles and sinks, a phenomenon occurring within an Arnold tongue [52, 55, 57]. Each time the Floquet multipliers of periodic orbits cross a root of unity, a pair of saddlenode bifurcation curves may be defined. These curves limit locally the corresponding 
resonance tongue. In addition, there are regions corresponding to homoclinic tangencies to dissipative periodic solutions, responsible for the persistence of Hénon-like strange attractors [52].

(3) Chaotic dynamics: the region above the graph of $h_{2}$ corresponds to vector fields whose flows exhibit rotational horseshoes [52] (see Appendix A.8). Theorem D of [52] may be seen as a criterion to obtain rotational horseshoes near $\Gamma$; once they develop, they persist for small perturbations.

As in [2, the graphs of $h_{1}$ and $h_{2}$ are not bifurcation lines; they define a region inside which the transitional dynamics occurs. From now on, without loss of generality, let us also assume that $\Phi(x)=\sin x, x \in \mathbb{S}^{1}$, which has exactly two critical points. It simplifies the computations and allows comparison with previous works. For $r \geq 3$, we denote by $\mathfrak{X}_{\mathrm{Byk}}^{r}\left(\mathbb{S}^{3}\right) \subset \mathfrak{X}^{r}\left(\mathbb{S}^{3}\right)$, the set of $C^{r}$-vector fields on $\mathbb{S}^{3}$ satisfying conditions (P1)-(P8b) and (P9).

\section{MAin RESUlts}

Let $\mathcal{T}$ be a neighborhood of the heteroclinic attractor $\Gamma$, which exists for $A=\lambda=0$ and $\omega \in \mathbb{R}^{+}$. For $\varepsilon>0$ small, define the set

$$
\mathcal{V}=\left\{\mu=(A, \lambda, \omega): \quad 0 \leq \lambda<A \leq \varepsilon \quad M \geq A+\lambda \quad \text { and } \quad \omega \in \mathbb{R}^{+}\right\}
$$

and let $\left(f_{\mu}\right)_{\mu \in \mathcal{V}}$ be a 3-parameter family of vector fields in $\mathfrak{X}_{\mathrm{Byk}}^{3}\left(\mathbb{S}^{3}\right)$. According to [52], there is $\tilde{\varepsilon}>0$ such that the first return map to a given global cross section $\Sigma$ to $\Gamma$ can be expressed, in local coordinates $(x, y) \in \Sigma$, by:

$$
\mathcal{F}_{\mu}(x, y)=\left[x-K \omega \ln (y+A+\lambda \sin x) \quad(\bmod 2 \pi),(y+A+\lambda \sin x)^{\delta}\right]+\ldots
$$

where

$$
(x, y) \in \mathcal{D}=\{x \in \mathbb{R} \quad(\bmod 2 \pi), \quad y / \tilde{\varepsilon} \in[-1,1] \quad \text { and } \quad y+A+\lambda \sin x>0\}
$$

and ... stand for small terms depending on $x$ and $y$ converging to zero along their derivatives. The main steps to get the expression (3.2) are revived in Section 4. Since $\delta \gtrsim 1$, for $A>0$ sufficiently small, the second component of $\mathcal{F}_{\mu}$ is contracting in $y$ (Remark 4.4).

Definition 2. Let $\left(x_{0}, y_{0}\right) \in \mathcal{D}$ and $\ell \in \mathbb{N}$. We say that $\left(x_{0}, y_{0}\right)$ is a $(1, \ell)$-fixed point of $\mathcal{F}_{\mu}$ if $\mathcal{F}_{\mu}\left(x_{0}, y_{0}\right)=\left(x_{0}+2 \ell \pi, y_{0}\right)$.

The main contribution of this article is the following result:

Theorem A. Let $f_{\mu} \in \mathfrak{X}_{\mathrm{Byk}}^{3}\left(\mathbb{S}^{3}\right)$ and $\ell \in \mathbb{N}$. There are two curves in the 3-dimensional parameter space $\mu=(A, \lambda, \omega) \in \mathcal{V}$ where $(1, \ell)$-fixed points of $\mathcal{F}_{\mu}$ undergo a discrete-time Bogdanov-Takens bifurcation. The points in the curves occur at values of $\omega=\omega_{\ell}^{\star}$.

The proof of Theorem $\mathrm{A}$ is performed in Subsection 5.3. after the statement of some preliminary technical results. Around the Bogdanov-Takens bifurcation, we found the following (secondary) codimension one bifurcations:

Corollary B. Let $f_{\mu} \in \mathfrak{X}_{\mathrm{Byk}}^{3}\left(\mathbb{S}^{3}\right)$. There are surfaces in the 3-dimensional parameter space $\mu=(A, \lambda, \omega) \in \mathcal{V}$ where $(1, \ell)$-fixed points of $\mathcal{F}_{\mu}$ undergo: 
(a) a saddle-node bifurcation;

(b) a Hopf bifurcation;

(c) generic (quadratic) homoclinic tangencies associated to a dissipative saddle poin 3 .

In the region between the surfaces corresponding to homoclinic tangencies, there is a transverse intersection of the stable and the unstable manifolds of an invariant saddle. The surfaces corresponding to Hopf and saddle-node bifurcations meet tangentially along a curve.

Around the transverse intersection of the manifolds, horseshoe dynamics occurs. Corollary B refines the findings of Sections 3.2 and 3.3 of [31], where the resonance wedges are projection of bifurcation surfaces. See also [48]. The 'necessity' of a Hopf bifurcation surface to explain the transition from an invariant torus to chaos has been raised in [46].

Strange attractors contribute to the richness and complexity of a dynamical system. SinaiBowen-Ruelle (SRB) measures represent visible statistical laws in non-uniformly hyperbolic systems. More details of these concepts may be found in Appendices A.5 and A.6. Chaos associated with them is both sustained in the space of parameters and observable. Next result ensures the existence of open regions in the parameter region (3.1) for which we observe strange attractors with SRB measures and historic behaviour (see Appendix A.4):

Corollary C. Let $f_{\mu} \in \mathfrak{X}_{\mathrm{Byk}}^{3}\left(\mathbb{S}^{3}\right)$. There is an open region in the space of parameters, say $\tilde{\mathcal{V}} \subset \mathcal{V}$, such that if $\mu \in \tilde{\mathcal{V}}$, then $\mathcal{F}_{\mu}$ exhibits:

(1) strange attractors with an ergodic SRB measure occuring in a subset of $\tilde{\mathcal{V}}$ with positive Lebesgue measure;

(2) an open set of initial conditions exhibiting historic behaviour occurring in a subset of $\tilde{\mathcal{V}}$ whose topological closure is $\tilde{\mathcal{V}} \mathbb{4}$

Taking advantage of the existence of the Hopf bifurcation surfaces, we may use the reasoning of Denjoy [22] to conclude the existence of a map $H$, arbitrarily $C^{1}$-close to $\mathcal{F}_{\mu}$, with a contracting non-trivial wandering domain. As defined in Appendix A.7, a wandering domain for $\mathcal{F}_{\mu}$ may be seen as a non-empty connected open set whose forward orbit is a sequence of pairwise disjoint open sets.

Corollary D. Let $f_{\mu} \in \mathfrak{X}_{\mathrm{Byk}}^{3}\left(\mathbb{S}^{3}\right)$. There is an open region in the space of parameters, say $\tilde{\mathcal{V}} \subset \mathcal{V}$, such that if $\mu \in \tilde{\mathcal{V}}$, then:

(1) $\mathcal{F}_{\mu}$ exhibits an attracting 2-dimensional torus, which is contractible5;

(2) there exists a diffeomorphism $H$ arbitrarily $C^{1}$-close to $\mathcal{F}_{\mu}$, exhibiting a contracting non-trivial wandering domain $D$ for which the union of the $\omega$-limit set of points in $D$ is a nonhyperbolic transitive Cantor set without periodic points.

\footnotetext{
${ }^{3} \mathrm{~A} \mathcal{F}_{\mu}$-fixed point $O$ is dissipative if $O$ is hyperbolic and $\left|\operatorname{det} \mathcal{F}_{\mu}(O)\right|<1$.

${ }^{4}$ The open set is defined in the phase space; the set $\tilde{\mathcal{V}}$ is defined in the space of parameters.

${ }^{5}$ The invariant circles, in the first return map, do not envelop the phase cylinder.
} 
The proofs of Corollaries B, $\mathrm{C}$ and $\mathrm{D}$ are performed in Sections 5.4, 5.5] and 5.6, respectively. In Section 6, we analyse the continuation of these bifurcations by studying the precise expressions of the eigenvalues of $D \mathcal{F}_{\mu}$ at the $(1, \ell)$-fixed points of $\mathcal{F}_{\mu}$. We derive an analytical expression for the Hopf and period-doubling bifurcations, as well as for the transitions node $\leftrightarrow$ focus. These plethora of bifurcations (among others), limited by two saddle-node bifurcation surfaces, is what we call a resonance wedge. An Arnold tongue may be seen as the projection of one of these wedges.

Remark 3.1. The Bogdanov-Takens bifurcation will be computed under the assumption that $M=A \pm \lambda$ (see Prop. 5.2). Since $A$ and $\lambda$ are small, then $M$ must be also small. This condition is achieved by assuming Hypothesis (P5): if $\delta \gtrsim 1$, then $M$ is small (cf. Figure 5 ).

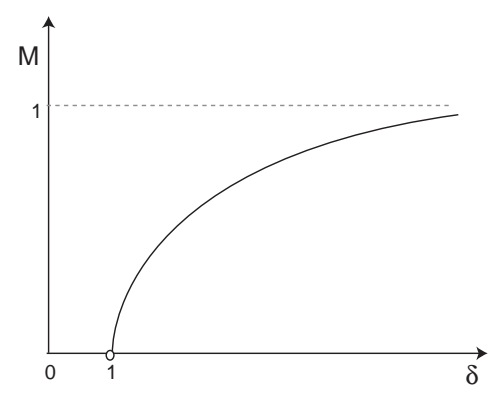

FiguRE 5. Graph of $M(\delta)=\delta^{\frac{1}{1-\delta}}-\delta^{\frac{\delta}{1-\delta}}$ for $\delta>1$.

\section{THE FIRST RETURN MAP}

We analyze the dynamics near $\Gamma$ through local maps, after selecting appropriate coordinates in neighborhoods of $\mathrm{O}_{1}$ and $\mathrm{O}_{2}$.

4.1. Local coordinates. In order to describe the dynamics around the cycles of $\Gamma$, we use the local coordinates near the equilibria $O_{1}$ and $O_{2}$ introduced in [44] (cf. [38, 52]). In these coordinates, we use cylindrical neighborhoods $V_{1}$ and $V_{2}$ in $\mathbb{R}^{3}$ of $O_{1}$ and $O_{2}$, respectively, of radius $\rho=\tilde{\varepsilon}>0$ and height $z=2 \tilde{\varepsilon}$. After a linear rescaling, we assume $\tilde{\varepsilon}=1$.

The boundaries of $V_{1}$ and $V_{2}$ consist of three components: the cylinder wall parametrised by $x \in \mathbb{R}(\bmod 2 \pi)$ and $|y| \leq 1$ with the cover

$$
(x, y) \mapsto(1, x, y)=(\rho, \theta, z)
$$

and two discs, the top and bottom of the cylinder. We consider polar coverings of these disks

$$
(r, \phi) \mapsto(r, \phi, \pm 1)=(\rho, \theta, z)
$$

where $0 \leq r \leq 1$ and $\varphi \in \mathbb{R}(\bmod 2 \pi)$. In $V_{1}$, we use the notation:

- $\operatorname{In}\left(O_{1}\right)$, the cylinder wall of $V_{1}$, consists of points that go inside $V_{1}$ in positive time;

- $\operatorname{Out}\left(O_{1}\right)$, the top and bottom of $V_{1}$, consists of points that go outside $V_{1}$ in positive time. 
We denote by $\operatorname{In}^{+}\left(O_{1}\right)$ the upper part of the cylinder, parametrised by $\left.\left.(x, y), y \in\right] 0,1\right]$ and by $\operatorname{In}^{-}\left(O_{1}\right)$ its lower part. The local stable manifold of $O_{1}, W_{\text {loc }}^{s}\left(O_{1}\right)$, corresponds to the circle parametrised by $y=0$. The cross-sections around $O_{2}$ are dual of the previous sections. The set $W_{\text {loc }}^{s}\left(O_{2}\right)$ corresponds to the intersection of the $z$-axis with the top and bottom of $V_{2}$; these two intersection points will be the origin of its coordinates. The set $W_{\text {loc }}^{u}\left(O_{2}\right)$ is defined by $y=0$ and:

- $\operatorname{In}\left(O_{2}\right)$, the top and bottom of $V_{2}$, consists of points that go inside $V_{2}$ in positive time;

- Out $\left(O_{2}\right)$, the cylinder wall of $V_{2}$, consists of points that go outside $V_{2}$ in positive time, with $\mathrm{Out}^{+}\left(\mathrm{O}_{2}\right)$ denoting its upper part, parametrised by $\left.\left.(x, y), y \in\right] 0,1\right]$ and Out $^{-}\left(\mathrm{O}_{2}\right)$ its lower part parametrised by $(x, y), x \in \mathbb{R}$ and $y \in[-1,0[$.

By construction, the flow is transverse to these cross-sections and the boundaries of $V_{1}$ and of $V_{2}$ may be written as the topological closure of $\operatorname{In}\left(O_{1}\right) \cup \operatorname{Out}\left(O_{1}\right)$ and $\operatorname{In}\left(O_{2}\right) \cup \operatorname{Out}\left(O_{2}\right)$, respectively.

Remark 4.1. The orientation of the angular coordinate near $\mathrm{O}_{2}$ is chosen to be compatible with the direction induced by the angular coordinate in $O_{1}$.

4.2. Local maps. Adapting [44, the trajectory of a point $(x, y) \in \operatorname{In}^{+}\left(O_{1}\right)$, leaves $V_{1}$ at $\operatorname{Out}\left(O_{1}\right)$ at

$$
\Phi_{1}(x, y)=\left(y^{\delta_{1}}+S_{1}(x, y ; A, \lambda, \omega), x-\frac{\omega \ln y}{E_{1}}+S_{2}(x, y ; A, \lambda, \omega)\right)=(r, \phi)
$$

where $\delta_{1}=\frac{C_{1}}{E_{1}}>1, S_{1}, S_{2}$ are smooth functions which depend on the parameters $A, \lambda$ and $\omega$ and satisfy:

$$
\left|\frac{\partial^{k+l+m}}{\partial x^{k} \partial y^{l} \partial A^{m_{1}} \partial \lambda^{m_{2}} \partial \omega^{m_{3}}} S_{i}(x, y ; A, \lambda, \omega)\right| \leq C y^{\delta_{1}+\sigma-l},
$$

where the numbers $C, \sigma$ are positive constants and $k, l, m_{1}, m_{2}, m_{3}$ are non-negative integers. In a similar way, a point $(r, \phi)$ in $\operatorname{In}\left(O_{2}\right) \backslash W_{\text {loc }}^{s}\left(O_{2}\right)$ leaves $V_{2}$ at $\operatorname{Out}\left(O_{2}\right)$ at

$$
\Phi_{2}(r, \phi)=\left(\phi-\frac{\omega \ln r}{E_{2}}+R_{1}(r, \phi ; A, \lambda, \omega), r^{\delta_{2}}+R_{2}(r, \phi ; A, \lambda, \omega)\right)=(x, y)
$$

where $\delta_{2}=\frac{C_{2}}{E_{2}}>1$ and $R_{1}, R_{2}$ satisfy a condition similar to (4.2). The expressions $S_{1}, S_{2}$, $R_{1}, R_{2}$ correspond to terms that vanish when $y$ and $r$ go to zero.

4.3. Global maps. The coordinates on $V_{1}$ and $V_{2}$ are chosen so that $\left[O_{1} \rightarrow O_{2}\right]$ connects points with $z>0$ (resp. $z<0$ ) in $V_{1}$ to points with $z>0$ (resp. $z<0$ ) in $V_{2}$. Points in $\operatorname{Out}\left(O_{1}\right) \backslash W_{\text {loc }}^{u}\left(O_{1}\right)$ near $W^{u}\left(O_{1}\right)$ are mapped into $\operatorname{In}\left(O_{2}\right)$ along a flow-box around each of the connections of $\left[\mathrm{O}_{1} \rightarrow \mathrm{O}_{2}\right]$. Assuming (P9), the transition

$$
\Psi_{1 \rightarrow 2}: \quad \operatorname{Out}\left(O_{1}\right) \quad \rightarrow \quad \operatorname{In}\left(O_{2}\right)
$$

does not depend neither on $\lambda, A$ nor $\omega$ and is the Identity map, a choice compatible with (P5) and (P7). Denote by $\eta$ the map:

$$
\eta=\Phi_{2} \circ \Psi_{1 \rightarrow 2} \circ \Phi_{1}: \quad \operatorname{In}\left(O_{1}\right) \backslash W_{\text {loc }}^{s}\left(O_{1}\right) \rightarrow \operatorname{Out}\left(O_{2}\right) .
$$


Omitting the higher order terms that appear in (4.1) and (4.3), for $y>0$ we may write:

$$
\eta(x, y)=\left(x-K \omega \ln y \quad(\bmod 2 \pi), y^{\delta}\right)
$$

with

$$
\delta=\delta_{1} \delta_{2} \gtrsim 1 \quad \text { and } \quad K=\frac{C_{1}+E_{2}}{E_{1} E_{2}}>0 .
$$

A similar expression is valid for $y<0$, after suitable changes. Using (P8) and (P9), for all $A>\lambda \geq 0$ and $\omega \in \mathbb{R}^{+}$, we may define the map $\Psi_{2 \rightarrow 1}: \operatorname{Out}\left(O_{2}\right) \rightarrow \operatorname{In}\left(O_{1}\right)$ that depends on the parameters $\lambda$ and $A$ (see Figure 6 ):

$$
\Psi_{2 \rightarrow 1}(x, y)=(x, y+A+\lambda \Phi(x)) \quad \text { where } \quad \Phi(x)=\sin x .
$$

Observe that $\Psi_{2 \rightarrow 1}$ does not depend on $\omega$. The expression of the first return map $\mathcal{F}_{\mu}$ follows

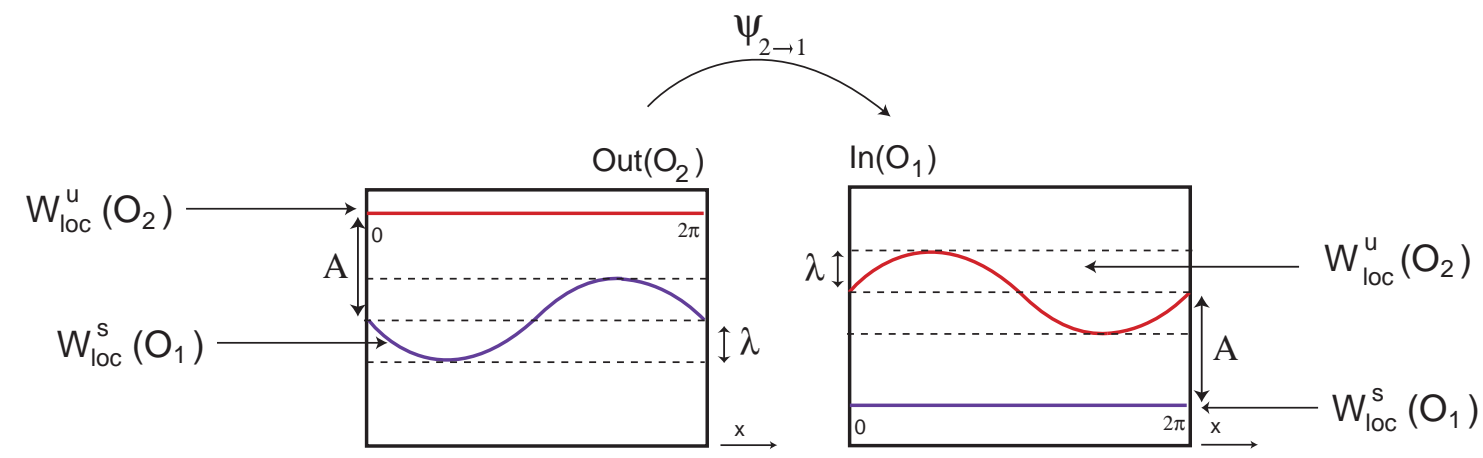

Figure 6. Geometry of the global map $\Psi_{2 \rightarrow 1}$. Scheme inspired by Gaspard 23 .

by composing the local and global maps constructed above. Let

$$
\mathcal{F}_{\mu}=\eta \circ \Psi_{2 \rightarrow 1}=\mathcal{D} \subset \operatorname{Out}\left(O_{2}\right) \quad \rightarrow \quad \mathcal{D} \subset \operatorname{Out}\left(O_{2}\right)
$$

be the first return map to $\operatorname{Out}\left(O_{2}\right)$, where $\mathcal{D} \neq \emptyset$ is the set of initial conditions $(x, y) \in$ $\operatorname{Out}\left(\mathrm{O}_{2}\right)$ whose solution returns to $\operatorname{Out}\left(\mathrm{O}_{2}\right)$. Composing $\eta$ (4.4) with $\Psi_{2 \rightarrow 1}$ (4.6), the expression of $\mathcal{F}_{\mu}$ is given by

$$
\begin{aligned}
\mathcal{F}_{\mu}(x, y) & =\left[x-K \omega \ln [y+A+\lambda \sin x] \quad(\bmod 2 \pi),(y+A+\lambda \sin x)^{\delta}\right] \\
& =:\left(\mathcal{F}_{1}^{\mu}(x, y), \mathcal{F}_{2}^{\mu}(x, y)\right) .
\end{aligned}
$$

The following remarks will be useful in the sequel.

Remark 4.2. The map $\mathcal{F}_{\mu}$ is $C^{3}$ and is well defined in a compact subset of $\operatorname{Out}\left(\mathrm{O}_{2}\right)$. Thus, results on circloid maps [45] may be applied to $\mathcal{F}_{\mu}$.

Remark 4.3. If $A=\lambda=0$ and $\omega \in \mathbb{R}^{+}$, then $\mathcal{F}_{1}^{(0,0, \omega)}(x, y)=x-K \omega \ln y$ may be identified with a rigid rotation on $\mathbb{S}^{1}=\mathbb{R} / 2 \pi$ and $\mathcal{F}_{2}^{(0,0, \omega)}(x, y)=y^{\delta}$ defines an invariant contracting foliation. 
Remark 4.4. Since $\delta>1$ and $1>\varepsilon>A>y \geq 0$, we may write:

$$
\left|\frac{\partial \mathcal{F}_{2}^{\mu}(x, y)}{\partial y}\right|=\left|\delta(y+A+\lambda \sin x)^{\delta-1}\right|=\mathcal{O}\left((A+\lambda)^{\delta-1}\right)<1,
$$

where $\mathcal{O}\left((A+\lambda)^{\delta-1}\right)$ represents the standard Landau notation. This means that, under Hypotheses (P1)-(P8b)-(P9), if $A>0$ small enough, then $\mathcal{F}_{2}^{\mu}$ is a contraction in the variable $y$.

\section{Proof of Theorem A And its Corollaries}

The main goal of this section is to prove Theorem $\mathrm{A}$ and its consequences. We start this task by giving preparatory results.

5.1. Fixed points of $\mathcal{F}_{\mu}$ and their stability. The $(1, \ell)$-fixed points of $\mathcal{F}_{\mu}$ in $\mathcal{D} \cap \operatorname{Out}\left(\mathrm{O}_{2}\right)$, say $p_{\ell}=\left(x_{\ell}, y_{\ell}\right) \in \operatorname{Out}\left(O_{2}\right), \ell \in \mathbb{N}$, are solutions of :

$$
\left\{\begin{array}{l}
x-K \omega \log (y+A+\lambda \sin x)=x+2 \ell \pi \\
(y+A+\lambda \sin x)^{\delta}=y .
\end{array}\right.
$$

Therefore,

$$
y_{\ell}+A+\lambda \sin x_{\ell}=\exp \left(\frac{-2 \ell \pi}{K \omega}\right) \quad \text { and } \quad y_{\ell}=\exp \left(\frac{-2 \ell \pi \delta}{K \omega}\right),
$$

which implies that

$$
A+\lambda \sin x_{\ell}=\exp \left(\frac{-2 \ell \pi}{K \omega}\right)-\exp \left(\frac{-2 \ell \delta \pi}{K \omega}\right) .
$$

For $\ell \in \mathbb{N}$, define the real-valued map

$$
G_{\ell}(\omega)=\exp \left(\frac{-2 \ell \pi}{K \omega}\right)-\exp \left(\frac{-2 \ell \delta \pi}{K \omega}\right), \quad \omega \in \mathbb{R}^{+} .
$$

whose graph is depicted in Figure 7, for different values of $\ell \in \mathbb{N}$. Observe that:

$$
A+\lambda \sin x_{\ell}=G_{\ell}(\omega) .
$$

The next result summarises some basic properties of $G_{\ell}$.

Lemma 5.1. For $\ell, \ell_{1}, \ell_{2} \in \mathbb{N}$, the following assertions are true:

(1) The map $G_{\ell}$ has a global maximum $M=\delta^{\frac{1}{1-\delta}}-\delta^{\frac{\delta}{1-\delta}}$ at $\omega_{\ell}^{\star}=\frac{2 \ell \pi(\delta-1)}{\ln \delta}>0$.

(2) The maximum of $G_{\ell}$ is independent of $\ell$.

(3) $\lim _{\omega \rightarrow 0^{+}} G_{\ell}(\omega)=\lim _{\omega \rightarrow+\infty} G_{\ell}(\omega)=0$.

(4) if $\ell_{1}<\ell_{2}$, then $\omega_{\ell_{1}}^{\star}<\omega_{\ell_{2}}^{\star}$. 


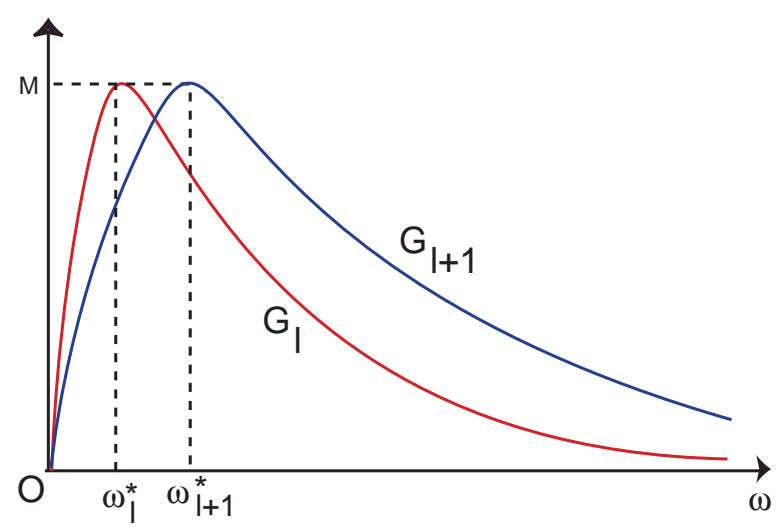

Figure 7. The map $G_{\ell}$ has a global maximum $M=\delta^{\frac{1}{1-\delta}}-\delta^{\frac{\delta}{1-\delta}}$ at $\omega_{\ell}^{\star}=$ $\frac{2 \ell \pi(\delta-1)}{\ln \delta}>0$.

Proof. Differentiating $G_{\ell}$ with respect to $\omega$ and multiplying by $K>0$, we get:

$$
\begin{aligned}
K G_{\ell}^{\prime}(\omega) & =\frac{2 \ell \pi}{\omega^{2}} \exp \left(\frac{-2 \ell \pi}{\omega}\right)-\frac{2 \ell \delta \pi}{\omega^{2}} \exp \left(\frac{-2 \ell \delta \pi}{\omega}\right) \\
& =\frac{2 \ell \pi}{\omega^{2}}\left[\exp \left(\frac{-2 \ell \pi}{\omega}\right)-\delta \exp \left(\frac{-2 \ell \delta \pi}{\omega}\right)\right] \\
& =\frac{2 \ell \pi}{\omega^{2}} \exp \left(\frac{-2 \ell \pi}{\omega}\right)\left[1-\delta \exp \left(\frac{-2 \ell(\delta-1) \pi}{\omega}\right)\right] .
\end{aligned}
$$

Since $\frac{2 \ell \pi}{\omega^{2}} \exp \left(\frac{-2 \ell \pi}{\omega}\right)>0$ for all $\omega \in \mathbb{R}^{+}$and $\ell \in \mathbb{N}$, we may conclude that:

$$
\begin{aligned}
G_{\ell}^{\prime}(\omega)=0 & \Leftrightarrow 1-\delta \exp \left(\frac{-2 \ell(\delta-1) \pi}{\omega}\right)=0 \\
& \Leftrightarrow \exp \left(\frac{-2 \ell(\delta-1) \pi}{\omega}\right)=1 / \delta \\
& \Leftrightarrow \frac{2 \ell(\delta-1) \pi}{\omega}=\ln \delta \\
& \Leftrightarrow \omega=\frac{2 \ell \pi(\delta-1)}{\ln \delta}=: \omega_{\ell}^{\star} .
\end{aligned}
$$

As suggested in Figure 7, it is easy to check that $G_{\ell}^{\prime}(\omega)>0$ if $\left.\omega \in\right] 0, \omega_{\ell}^{\star}\left[\right.$ and $G_{\ell}^{\prime}(\omega)<0$ otherwise. This implies that $G_{\ell}$ is increasing in $] 0, \omega_{\ell}^{\star}[$ and decreasing in $] \omega_{\ell}^{\star}, \infty[$. Furthermore, 


$$
\begin{aligned}
G_{\ell}\left(\omega_{\ell}^{\star}\right) & =\exp \left(\frac{-2 \ell \pi \ln \delta}{2 \ell \pi(\delta-1)}\right)-\exp \left(\frac{-2 \ell \delta \pi \ln \delta}{2 \ell(\delta-1) \pi}\right) \\
& =\exp \left(\frac{\ln \delta}{1-\delta}\right)-\exp \left(\frac{\delta \ln \delta}{1-\delta}\right) \\
& =\delta^{\frac{1}{1-\delta}}-\delta^{\frac{\delta}{1-\delta}}=: M .
\end{aligned}
$$

The following two limits are zero as a result of the analytic expression of $G_{\ell}$ :

$$
\lim _{\omega \rightarrow 0^{+}}\left[\exp \left(\frac{2 \ell \pi}{K \omega}\right)-\exp \left(\frac{2 \ell \delta \pi}{K \omega}\right)\right]=0=\lim _{\omega \rightarrow+\infty}\left[\exp \left(\frac{2 \ell \pi}{K \omega}\right)-\exp \left(\frac{2 \ell \delta \pi}{K \omega}\right)\right] .
$$

The last assertion follows straightforwardly from the expression of $\omega_{\ell}^{\star}$.

In order to determine the Lyapunov stability of the fixed points of $\mathcal{F}_{\mu}$, we compute the derivative of $\mathcal{F}_{\mu}$, at a general $(1, \ell)$-fixed point $\left(x_{\ell}, y_{\ell}\right)$.

$$
D \mathcal{F}_{\mu}\left(x_{\ell}, y_{\ell}\right)=\left(\begin{array}{cr}
1-\frac{K \omega \lambda \cos x_{\ell}}{y_{\ell}+A+\lambda \sin x_{\ell}} & -\frac{K \omega}{y_{\ell}+A+\lambda \sin x_{\ell}} \\
\lambda \delta\left(y_{\ell}+A+\lambda \sin x_{\ell}\right)^{\delta-1} \cos x_{\ell} & \delta\left(y_{\ell}+A+\lambda \sin x_{\ell}\right)^{\delta-1}
\end{array}\right)
$$

In order to find the $(1, \ell)$-fixed points of $\mathcal{F}_{\mu}$, we need to solve the equation:

$$
\varphi(x)=G_{\ell}(\omega), \quad \text { where } \quad \varphi(x)=A+\lambda \sin x .
$$

For $A>\lambda>0$ fixed and $\omega \in \mathbb{R}^{+}$, the graph of the left hand side of (5.4), say $\varphi(x)$, depends on $x \in[0,2 \pi]$ and does not depend on $\omega$. On the other hand, the graph of the right hand side of (5.4) does not depend on $x$.

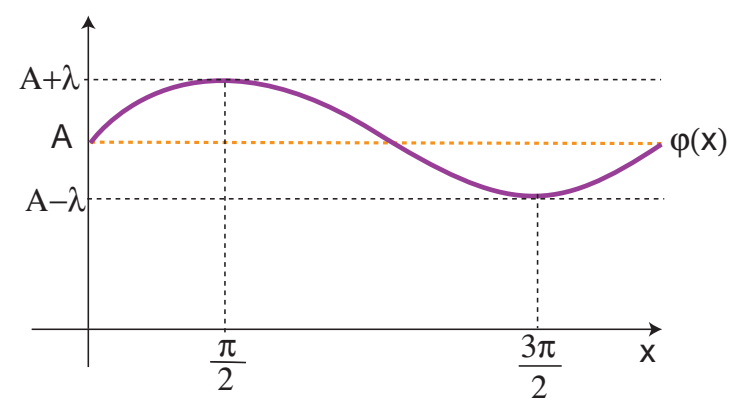

Figure 8. Graph of $\varphi(x)=A+\lambda \sin x, x \in[0,2 \pi]$.

As illustrated in Figures 8 and 9, finding $(1, \ell)$-fixed points of $\mathcal{F}_{\mu}$ amounts to intersect the graph of $\varphi(x)$ with a horizontal line. The line moves first up and then down, as $\omega$ increases. Since the range of $\varphi$ is the interval $[A-\lambda, A+\lambda]$, and the range of $G_{\ell}(\omega)$ is the interval $(0, M]$, the geometry of the solution set depends on the relative positions of these intervals. 
From now on, we use the inequality $M \geq A+\lambda, A \in[0, \varepsilon]$ - see (3.1). The analytic treatment of the other cases are similar to the approach of Section 5 of [39].

As $\omega$ increases from 0 , there is a threshold value $\omega_{1}$ for which the horizontal line at height $G_{\ell}\left(\omega_{1}\right)$ touches the graph of $\varphi$ at $x=3 \pi / 2$. At this point we have $\sin (x)=-1$. As $\omega$ increases further, each tangency unfolds as two intersection points of the graph with the horizontal line. There is a saddle-node at the points $\left(x^{(1)}, G_{\ell}\left(\omega_{1}\right)\right)=\left(3 \pi / 2, G_{\ell}\left(\omega_{1}\right)\right)$, as we will see in Proposition 5.2. The surface $G_{\ell}(\omega)=A \pm \lambda$ defines the boundaries of the $(1, \ell)$-resonance wedge. The horizontal line may move further up and a pair of solutions come together at a second saddle-node at $\left.\left(x^{(2)}, G_{\ell}\left(\omega_{2}\right)\right)\right)=\left(\pi / 2, G_{\ell}\left(\omega_{2}\right)\right)$ and reappear at a saddle-node at $\left(x^{(3)}, G_{\ell}\left(\omega_{3}\right)\right)=\left(\pi / 2, G_{\ell}\left(\omega_{3}\right)\right)$ coming together finally at $\left(x^{(4)}, G_{\ell}\left(\omega_{4}\right)\right)=\left(3 \pi / 2, G_{\ell}\left(\omega_{4}\right)\right)$. The evolution of the geometry of solutions of (5.4), as $\omega$ varies, is illustrated on the right side of Figure 9. We show below that, at these points, the map $D \mathcal{F}_{\mu}$ has an eigenvalue equal to 1.

Based on (5.1), for each $\ell \in \mathbb{N}$, define the map

$$
y(\omega)=\exp \left(\frac{-2 \ell \pi \delta}{K \omega}\right), \quad \text { with } \quad \omega \in \mathbb{R}^{+} .
$$

5.2. Double eigenvalue 1. A discrete-time Bogdanov-Takens bifurcation occurs when the maximum of $G_{\ell}$ coincides with either the minimum or the maximum of $\varphi$. In this subsection, we check the necessary linear conditions for this bifurcation. In this section, we implicitly use the fact that the network is weakly attracting (see Remark 3.1).

Proposition 5.2. For $G_{\ell}\left(\omega_{\ell}^{\star}\right)=A \pm \lambda$, the derivative $D \mathcal{F}_{\mu}\left(x^{(N)}, y\left(\omega_{\ell}^{\star}\right)\right)$ at a solution of (5.4) has 1 as a double eigenvalue and is not the identity, for $N=1, \ldots, 4$.
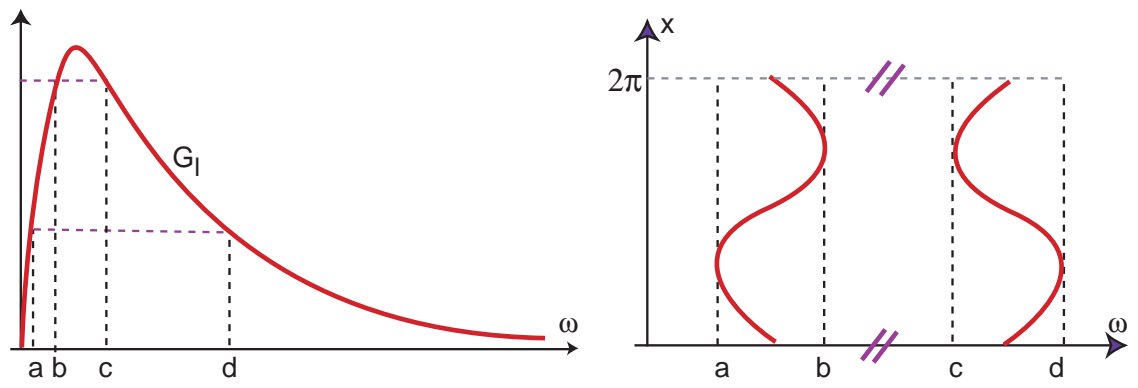

Figure 9. Graph of $G_{\ell}$ and evolution of the fixed points when $\omega$ varies. $a=\omega_{1}$, $b=\omega_{2}, c=\omega_{3}$ and $d=\omega_{4}$. Double bars mean that the sides are identified.

Proof. Computing the derivative $D \mathcal{F}_{\mu}$ at the points $\left(x^{(N)}, y\left(\omega_{N}\right)\right), N=1, \ldots, 4$, where $\sin \left(x_{N}\right)= \pm 1$, we get:

$$
D \mathcal{F}_{\mu}\left(x^{(N)}, y\left(\omega_{N}\right)\right)=\left(\begin{array}{cr}
1 & -\frac{K \omega}{y\left(\omega_{N}\right)+A \pm \lambda} \\
0 & \delta\left(y\left(\omega_{N}\right)+A \pm \lambda\right)^{\delta-1}
\end{array}\right)
$$

At $\left(x^{(N)}, y\left(\omega_{N}\right)\right)$ the Jacobian matrix is triangular and so the two eigenvalues are

$$
\Delta_{1}=1 \quad \text { and } \quad \Delta_{2}=\delta\left(y\left(\omega_{N}\right)+A \pm \lambda\right)^{\delta-1}>0 .
$$


Since $\omega_{\ell}^{\star}$ was defined to be the value of $\omega$ where the function $G_{\ell}$ defined in (5.3) has a global maximum, then $\frac{d G_{\ell}}{d t}\left(\omega_{\ell}^{\star}\right)=0$. In particular,

$$
\begin{aligned}
\Delta_{2} & =\delta\left(y\left(\omega_{\ell}^{\star}\right)+A \pm \lambda\right)^{\delta-1} \\
& =\delta\left(\exp \left(\frac{-2 \ell \pi}{\omega_{\ell}^{\star}}\right)\right)^{\delta-1} \\
& =\delta\left(\exp \left(\frac{-2 \ell \pi(\delta-1)}{\omega_{\ell}^{\star}}\right)\right) \\
& =\delta\left(\exp \left(\frac{-2 \ell \pi(\delta-1) \ln \delta}{2 \ell \pi(\delta-1)}\right)\right) \\
& =\delta \exp \left(\ln \delta^{-1}\right)=1 .
\end{aligned}
$$

Hence the derivative $D \mathcal{F}_{\mu}$, at the solutions of (5.4) with $G_{\ell}\left(\omega_{\ell}^{\star}\right)=A \pm \lambda$, has a double eigenvalue equal to 1 , and is not the identity as we may confirm in (5.5).

5.3. Proof of Theorem $\mathbf{A}$. Proposition 5.2 indicates a (possible) bifurcation of codimension 2 , corresponding to a curve in the 3 -dimensional parameter space $\mu=(A, \lambda, \omega)$, where we expect to find a discrete-time Bogdanov-Takens bifurcation. This bifurcation occurs at points where 1 is a double eigenvalue, the derivative is not the identity and the map $\mathcal{F}_{\mu}$ satisfies a finite number of non-degeneracy conditions. In this section, we check these nonlinear conditions. We recall the main ideas of [16, 58] adapted to our purposes.

Along the surface defined by $\pm \lambda=A-G_{\ell}\left(\omega_{\ell}^{\star}\right)$, the map $\mathcal{F}_{\mu}$ has a fixed point $p_{\ell}=$ $\left(x^{(N)}, y\left(\omega_{\ell}^{\star}\right)\right), N=1, \ldots, 4$ and $\ell \in \mathbb{N}$, such that $D \mathcal{F}_{\mu}\left(p_{\ell}\right)$ has a double unit eigenvalue but is not the identity.

For $\lambda=\lambda_{0}>0$ fixed, by composing the translation $\left(x_{\ell}, y_{\ell}\right) \mapsto(0,0)$ with the following (local) change of coordinates:

$$
(x, y) \mapsto(x, C y) \quad \text { where } \quad C=\frac{-2 K \ell \pi(\delta-1)}{\delta^{\frac{\delta}{1-\delta}} \ln \delta}<0,
$$

for $\nu=\left(A, \lambda_{0}, \omega\right)$ near $\left(G_{\ell}\left(\omega_{\ell}^{\star}\right) \mp \lambda_{0}, \lambda_{0}, \omega_{\ell}^{\star}\right) \in \mathcal{V}$, the map $D \mathcal{F}_{\nu}(0,0)$ has the form:

$$
D \mathcal{F}_{\nu}(x, y)=\left(\begin{array}{cc}
1 & 1 \\
0 & 1
\end{array}\right)\left(\begin{array}{l}
x \\
y
\end{array}\right)+\left(\begin{array}{c}
a(x, y ; \nu) \\
b(x, y ; \nu)
\end{array}\right)+\mathcal{O}\left(\|(x, y)\|^{3}\right)
$$

where the polynomial expansions of order 2 of $a$ and $b$ may be written as:

$$
a(x, y ; \nu)=a_{00}(\nu)+a_{10}(\nu) x+a_{01}(\nu) y+\frac{1}{2} a_{20}(\nu) x^{2}+a_{11}(\nu) x y+\frac{1}{2} a_{02}(\nu) y^{2}
$$

and

$$
b(x, y ; \nu)=b_{00}(\nu)+b_{10}(\nu) x+b_{01}(\nu) y+\frac{1}{2} b_{20}(\nu) x^{2}+b_{11}(\nu) x y+\frac{1}{2} b_{02}(\nu) y^{2}
$$




\begin{tabular}{|c|c|c|}
\hline Coefficient & $x=\frac{\pi}{2}$ & $x=\frac{3 \pi}{2}$ \\
\hline \hline$a_{20}(\nu)$ & $\frac{C K \omega \lambda}{(A-\lambda)^{2}}<0$ & $-\frac{C K \omega \lambda}{(A+\lambda)^{2}}>0$ \\
\hline \hline$b_{11}(\nu)$ & $-(A-\lambda)^{\delta-2} \lambda \delta(1-\delta)>0$ & $(A+\lambda)^{\delta-2} \lambda \delta(1-\delta)<0$ \\
\hline \hline$b_{20}(\nu)$ & $-(A-\lambda)^{\delta-2} \lambda \delta(1-\delta)>0$ & $(A+\lambda)^{\delta-2} \lambda \delta(1-\delta)<0$ \\
\hline
\end{tabular}

TABLE 1. Leading coefficients $a_{20}(\nu), b_{11}(\nu)$ and $b_{20}(\nu)$ of (5.9) used to check the nonlinear conditions for the discrete-time Bogdanov-Takens. Note that $b_{11}(\nu)=$ $b_{20}(\nu)$, for $\nu=\left(A, \lambda_{0}, \omega\right)$ near $\left(G_{\ell}\left(\omega_{\ell}^{\star}\right) \mp \lambda_{0}, \lambda_{0}, \omega_{\ell}^{\star}\right)$.

with

$$
a_{00}(0,0)=a_{10}(0,0)=a_{01}(0,0)=b_{00}(0,0)=b_{10}(0,0)=b_{01}(0,0)=0 .
$$

The leading coefficients of $a$ and $b$ that will be used in the sequel are listed in Table 1 . We concentrate our attention on the fixed point associated to $x=3 \pi / 2$; the other is similar. By Proposition 3.1 of Yagasaki [58], since

$$
b_{20}(\nu)=(A+\lambda)^{\delta-2} \lambda \delta(1-\delta)<0 \quad \text { for } \quad \delta>1,
$$

and

$$
a_{20}(\nu)+b_{11}(\nu)-b_{20}(\nu)=-\frac{C K \omega \lambda}{(A+\lambda)^{2}}=a_{20}>0
$$

then there exists a bifurcation point of codimension 2 at $\left(x_{N}, y\left(\omega_{\ell}^{\star}\right)\right)$ with $N=1, \ldots, 4$ and $\ell \in \mathbb{N}$ such that, nearby (see Figure [10):

(1) there exist two curves associated to saddle-node bifurcation ( $\mathbf{s n}_{1}$ and $\left.\mathbf{s n}_{2}\right)$;

(2) there exists one curve associated to a Hopf bifurcation at the stable focus born at the saddle-node bifurcation (hopf);

(3) there exists a region with a Lyapunov stable invariant circle created at the Hopf bifurcation, since

$$
b_{20}\left(a_{20}+b_{11}-b_{20}\right)<0
$$

(all coefficients are computed at $\left.\left(G_{\ell}\left(\omega_{\ell}^{\star}\right) \mp \lambda_{0}, \lambda_{0}, \omega_{\ell}^{\star}\right)\right)$;

(4) there exist two curves, $\mathbf{h}_{1}$ and $\mathbf{h}_{2}$, associated to a homoclinic bifurcation where the stable and unstable manifolds of the saddle point born at (1) touch tangencially. The distance between the two homoclinic bifurcation curves is exponentially small with 
(1)

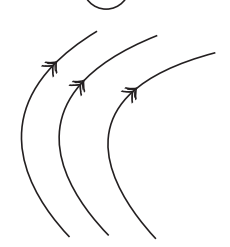

(2)
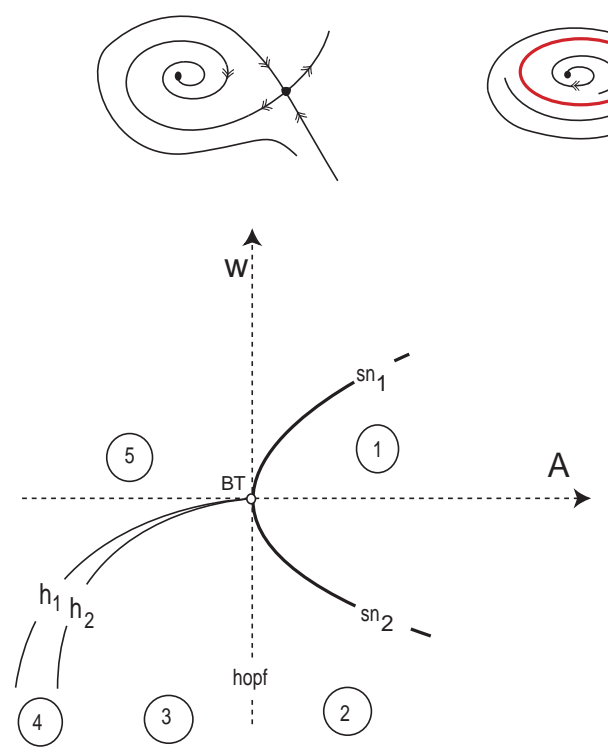

(3)
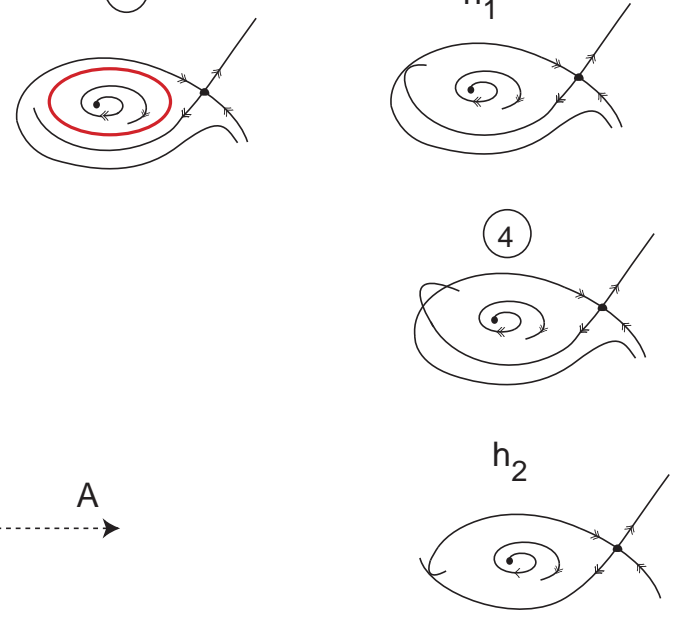

(5)

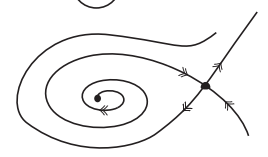

Figure 10. Dynamics for the discrete-time Bogdanov-Takens bifurcation in $(A, \omega)$, after a smooth change of coordinates. $\mathbf{s n}_{1}$ and $\mathbf{s n}_{2}$ : Saddle-node bifurcations; hopf: Hopf bifurcation; $\mathbf{h}_{1}$ and $\mathbf{h}_{2}$ : homoclinic tangencies associated to a dissipative fixed point. 1: no recurrent dynamics; 2 : the unstable manifold of the saddle is connected with the stable manifold of the focus; 3 : saddle and a stable periodic orbit; 4 : horseshoe dynamics; 5 : the unstable manifold of the focus intersects the stable manifold of the saddle.

respect to $\sqrt{\left.\left\|\nu-\left(G_{\ell}\left(\omega_{\ell}^{\star}\right) \mp \lambda_{0}, \omega_{\ell}^{\star}\right)\right\|\right)}$;

(5) the invariant manifolds of a dissipative saddle intersect transversely inside the parameter region between the curves $\mathbf{h}_{1}$ and $\mathbf{h}_{2}$ and do not intersect outside it.

Remark 5.3. In Proposition 3.1 of [58], there exists an extra condition: $\operatorname{det} D_{\mu} \nu(0) \neq 0$. This inequality serves to describe (in some system of coordinates) the explicit expression for the bifurcating curves, which is used to conclude that the hopf bifurcation curve is tangent to the saddle-node bifurcation curves $\mathbf{s n}_{1}$ and $\mathbf{s n}_{2}$, at the bifurcation point.

For $\ell \in \mathbb{N}$, denote by $\mathbf{B} \mathbf{T}_{\ell}^{1}$ and $\mathbf{B} \mathbf{T}_{\ell}^{2}$ the two discrete-time Bogdanov-Takens bifurcation in the bifurcation parameter $(A, \lambda)$ such that $A>\lambda=\lambda_{0}$ which occur for $\omega=\omega_{\ell}^{\star}$ :

$$
\mathbf{B T}_{\ell}^{1} \mapsto A=G_{\ell}\left(\omega_{\ell}^{\star}\right)-\lambda, \quad \mathbf{B T}_{\ell}^{2} \mapsto A=G_{\ell}\left(\omega_{\ell}^{\star}\right)+\lambda .
$$

5.4. Proof of Corollary B. This is a direct corollary of Theorem A, For $\lambda=\lambda_{0}>0$ and $\ell \in \mathbb{N}$ fixed, there exist two points of Bogdanov-Takens bifurcation for the map $\mathcal{F}_{\mu}$ at $p_{\ell}$ : $\mathbf{B} \mathbf{T}_{\ell}^{1}$ and $\mathbf{B} \mathbf{T}_{\ell}^{2}$ (see (5.11)). As depicted in Figure 12, varying smoothly $\lambda \gtrsim 0$ around each Bogdanov-Takens bifurcation: 
(a) there exist two surfaces of saddle-node bifurcations $\left(\mathbf{S N}_{\ell}^{1}\right.$ and $\left.\mathbf{S N}_{\ell}^{2}\right)$;

(b) there exists a surface of Hopf bifurcations $\left(\mathbf{H o p f}_{\ell}\right)$;

(c) there exist two surfaces of homoclinic tangencies $\left(\mathbf{H}_{\ell}^{1}\right.$ and $\left.\mathbf{H}_{\ell}^{2}\right)$.

The two surfaces (c) correspond to bifurcations at which the stable and unstable manifolds of a dissipative saddle point are tangent. In the region between these surfaces there is a transverse intersection of the stable and the unstable manifolds of a saddle. This configuration implies that the dynamics of $\mathcal{F}_{\mu}$ is equivalent to Smale's horseshoe. Last assertion of Corollary B follows from Remark 5.3 .

Remark 5.4. We cannot exclude the possibility that the two surfaces $\mathbf{H}_{\ell}^{1}$ and $\mathbf{H}_{\ell}^{2}, \ell \in \mathbb{N}$, coincide, although it would be a highly non-generic behaviour.

5.5. Proof of Corollary C. The existence of $\mathbf{H}_{\ell}^{1}$ and $\mathbf{H}_{\ell}^{2}, \ell \in \mathbb{N}$, shows that there are surfaces in the space of parameters for which the map $\mathcal{F}_{\mu}$ has a quadratic (generic) homoclinic tangency associated to a dissipative periodic point. Using [41], there exists a positive measure set $\Delta$ of parameter values, so that for every $\mu \in \Delta \subset \mathcal{V}, \mathcal{F}_{\mu}$ admits a strange attractor of Hénon-type with an ergodic SRB measure. The existence of historic behaviour is a combination of the latter tangencies and Theorem A of Kiriki and Soma [34].

5.6. Proof of Corollary D. The first part of the corollary is a straightforward consequence of the Hopf bifurcation surface of Corollary B, from which a stable torus emerge (see (5.10) ). The proof for the second part is a simple inspection of Theorem B of [51] (see also references therein). For the sake of completeness, we list the main steps of the proof:

(1) for each $\lambda=\lambda_{0}>0$, we write explicitly the normal form for the family of Hopf bifurcation, which creates an attracting invariant circle;

(2) perturb (if necessary) the truncated normal form in order to obtain an irrational rotation on the circle, say $H_{1}$;

(3) perturb $H_{1}$, using Denjoy procedure [22], in order to obtain contracting wandering domains. The resulting map is $C^{1}$-close to $\mathcal{F}_{\mu}, \mu \in \mathcal{V} \cap \mathbf{H o p f}_{\ell}, \ell \in \mathbb{N}$.

\section{Dissecting a Resonance Wedge: Putting All together}

Theorem \$ may be seen as a "local" theorem. A new problem arises: how the surfaces of bifurcations of Corollary B are globally organised? This section provides a partial answer to this question. We plot the graphs of the maps that defines the Hopf, the transitions node $\leftrightarrow$ focus and the period-doubling bifurcation, as function of the parameters $\mu=(A, \lambda, \omega) \in \mathcal{V}$. These bifurcations arise in a form consistent with Corollary B.

6.1. Necessary conditions for bifurcations. The Hopf surfaces are particularly significant, as they separate the resonance regions into parts with an attracting periodic orbit and parts with none. To simplify the notation, denote by det and trace the determinant and the 
trace of $D \mathcal{F}_{\mu}(x, y)($ see $(5.5))$, respectively.

$$
\begin{aligned}
\operatorname{det}:=\operatorname{det} D \mathcal{F}_{\mu}(x, y) & =\delta(y+A+\lambda \sin x)^{\delta-1} \\
\text { trace }:=\operatorname{trace} D \mathcal{F}_{\mu}(x, y) & =1-\frac{K \omega \lambda \cos x}{y+A+\lambda \sin x}+\delta(y+A+\lambda \sin x)^{\delta-1} .
\end{aligned}
$$

Note that for a $2 \times 2$-real matrix $D \mathcal{F}_{\mu}(x, y)$, its eigenvalues are the roots of the polynomial in $t$ given by

$$
P(t)=t^{2}-\operatorname{trace} t+\operatorname{det}, \quad \text { say } \quad t=\frac{\operatorname{trace} \pm \sqrt{\operatorname{trace}^{2}-4 \mathrm{det}}}{2} .
$$

Up to nonlinear conditions, a Hopf bifurcation (for a map) occurs when the norm of the complex (conjugate) eigenvalues crosses the unit circle. This happens when

$$
\operatorname{det}=1 \quad \text { and } \quad \text { trace } \in]-2,2[\text {. }
$$

We know that $G_{\ell}(\omega)=\varphi(x)$ because $(x, y)$ is a $(1, \ell)$-fixed point of $\mathcal{F}_{\mu}$ - see (5.4). This means that

$$
\begin{aligned}
& \exp \left(\frac{-2 \ell \pi}{K \omega}\right)-\exp \left(\frac{-2 \ell \delta \pi}{K \omega}\right)=A+\lambda \sin x \\
\Leftrightarrow & G_{\ell}(\omega)-A=\lambda \sin x .
\end{aligned}
$$

Since $\lambda \cos x= \pm \sqrt{\lambda^{2}-\lambda^{2} \sin x}= \pm \sqrt{\lambda^{2}-\left(G_{\ell}(\omega)-A\right)^{2}}$, we may write:

$$
\begin{aligned}
\text { trace } & =1-\frac{K \omega \lambda \cos x}{y+A+\lambda \sin x}+\delta(y+A+\lambda \sin x)^{\delta-1} \\
& =1 \mp \frac{K \omega \sqrt{\lambda^{2}-\left(G_{\ell}(\omega)-A\right)^{2}}}{\exp \left(\frac{-2 \ell \pi}{K \omega}\right)}+\delta \exp \left(\frac{-2 \ell(\delta-1) \pi}{K \omega}\right) \\
\text { det } & =\delta(y+A+\lambda \sin x)^{\delta-1}=\delta \exp \left(\frac{-2 \ell(\delta-1) \pi}{K \omega}\right) .
\end{aligned}
$$

In Figure 11, for $\ell=K=2, \delta=3$, we have plotted of the following surfaces, in the 3 -parameter space $\mathcal{V}$ :

(1) SN: the saddle-nodes bifurcations corresponding to $G_{\ell}(\omega)=A \pm \lambda$;

(2) Hopf: the Hopf bifurcations corresponding to trace $\in]-2,2[$ and det $=1$;

(3) $\mathbf{N F} / \mathbf{F N}$ : the transitions node $\leftrightarrow$ focus corresponding to $\operatorname{trace}^{2}-4 \operatorname{det}=0$;

(4) PD: the period-doubling bifurcation corresponding to the case where one root of $P(t)$ is -1 . 
(a)

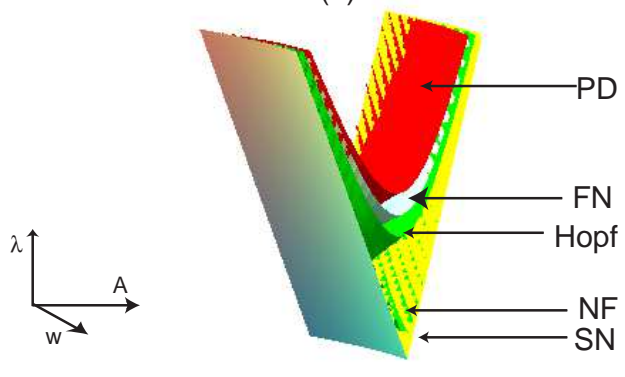

(b)

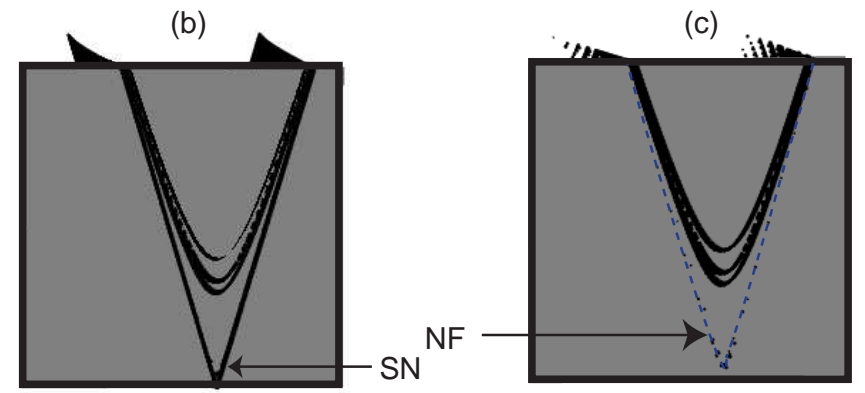

Figure 11. Graphs of det $=1$ and trace $\in]-2,2\left[(\right.$ Hopf $)$, trace $^{2}-4 \operatorname{det}=0$ $(\mathbf{N F}$ and $\mathbf{F N}), G_{\ell}(\omega)=A \pm \lambda(\mathbf{S N})$ and one of the roots of $P(t)$ equals to $-1(\mathbf{P D})$, with $\ell=K=1, \delta=3$, numerically plotted using Maple, $A \in[0 ; 0.5], \lambda \in[0 ; 0.1]$, $\omega \in[0.5,10]$. In (c) the $\mathbf{S N}$ surfaces have not been plotted. The $\mathbf{S N}$ and $\mathbf{N F}$ surfaces are almost indistinguishable in the gray section $(\omega=10)$.

Numerical plots suggest that the surface Hopf $_{\ell}$ connects both "local" Hopf surfaces that appear near $\mathbf{B} \mathbf{T}_{\ell}^{1}$ and $\mathbf{B} \mathbf{T}_{\ell}^{2}$ (see Corollary B] and Figure $12(\mathrm{a})$ ). In the plane defined by $\omega=10$ of Figure 11, the curves corresponding to NF and SN are very close; in (b) of Figure 11, we cannot distinguish them.

Remark 6.1. On the PD surface, one of the multipliers is equal to -1 and, in the $\mathbf{B T}$ curve, both multipliers are equal to +1 . Therefore, the loci associated to these two bifurcations (for the same fixed point) should never meet. However, as suggested by Figure12, restricted to the plane defined by $\lambda=0.1$, the $\mathbf{P D}$ curve "terminates" very close to the $\mathbf{B T}$ bifurcation points. We conjecture that it "terminates" within the region limited by $\mathbf{h}_{1}$ and $\mathbf{h}_{2}$ (homoclinics associated to the discrete BT bifurcation) due to horseshoe formation/destruction.

Remark 6.2. The theory of the previous section has been performed for $(1, \ell)$-fixed points of $\mathcal{F}_{\mu}$. However, all these phenomena can also be observed for wedges associated to other rotation number; of course, the analytic expressions for the bifurcation surfaces are different.

6.2. Summarizing movie. In this subsection, we give a heuristic discussion of what is going on within an Arnold wedge. We compare our results with those found in previous works by other authors; all results agree well with the theoretical information of [43, 55].

For $\varepsilon>0$ small, the choice of parameters in Section 2 allows us to build the bifurcation diagram of Figure 13 in $\mathcal{V}$ (see (3.1)). Dynamical bifurcation surfaces in resonance wedge may be projected into a generic plane, giving rise to what the literature calls an Arnold tongue. This projection is sketched on the right side of Figure 13 and Figure 14. All resonant wedges have the origin as a common point.

For $A>\lambda \geq 0$ fixed, if $\omega$ is sufficiently small, the flow of (2.1) exhibits a 2-dimensional non-contractible torus which is globally attracting and normally hyperbolic. The dynamics of $\mathcal{F}_{\mu}$ is governed by the dynamics of a circle map. There is a positive measure set $\Delta \subset \mathcal{V}$ so that the rotation number of $\mathcal{F}_{\mu}$ is irrational if and only if $\mu \in \Delta$ [27, 28]. 
(a)

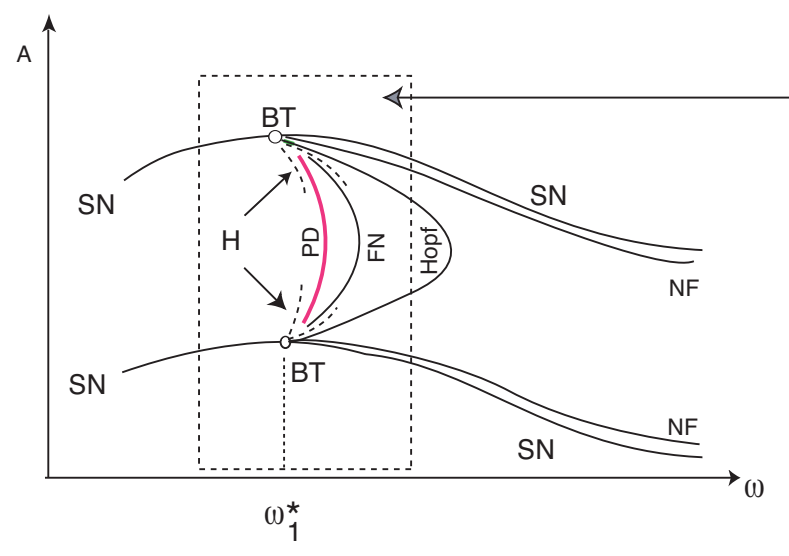

(c)

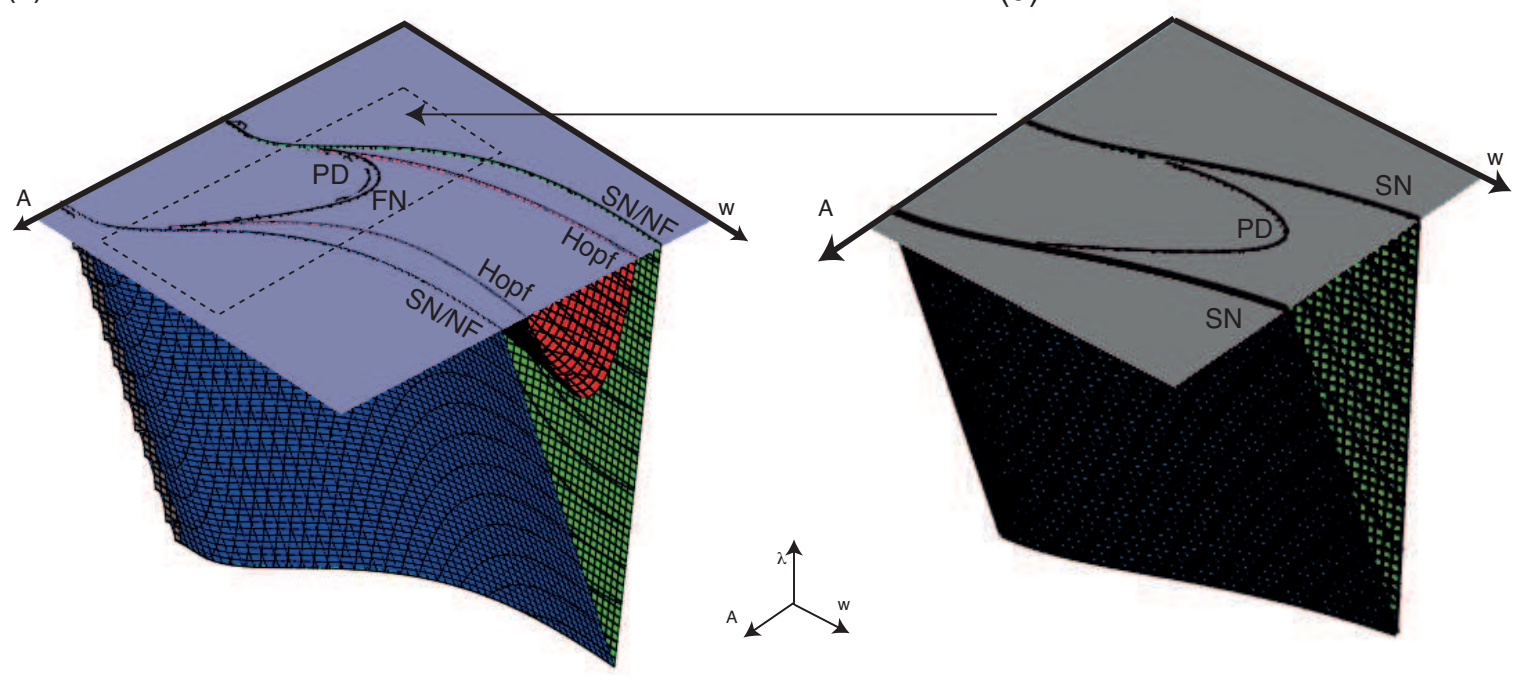

(b)

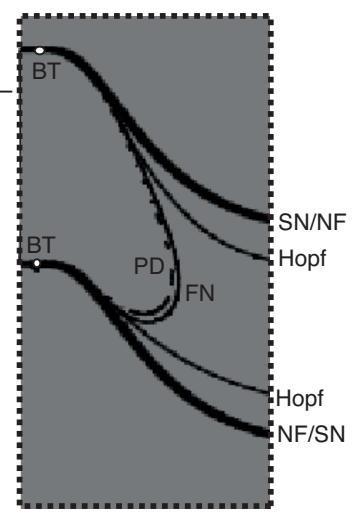

(d)

Figure 12. (a) Plausible bifurcation diagram in the plane $(\omega, A)$, for $\lambda=\lambda_{0}>0$ and $\ell=K=1$. (a): theoretical scheme; (b) numerical scheme using Maple with $\ell=K=1, \delta=3, A \in[0 ; 0.5], \lambda=0.1, \omega \in[2,10]$. (c) Approximate bifurcation diagram in the space $(A, \lambda, \omega)$, for $\ell=K=1, \delta=3, A \in[0 ; 0.5], \lambda \in[0 ; 0.1]$, $\omega \in[1,30]$. (d) Approximate bifurcation diagram in the space $(A, \lambda, \omega)$, for $\lambda=0.1$ and $\ell=K=1, \delta=3, A \in[0.1 ; 0.4], \lambda \in[0 ; 0.1], \omega \in[2,10]$. The gray plane corresponds to $\lambda=0.1$. Bifurcations: BT: Bogdanov-Takens, SN: saddle-node, H: homoclinic tangencies, FN/NF: transitions focus $\leftrightarrow$ node, Hopf: Hopf, PD: period-doubling. In (d), just the surfaces $\mathbf{S N}$ and $\mathbf{P D}$ have been plotted.

Within $\mathcal{V}$, for each $\ell \in \mathbb{N}$, we may define a resonance wedge, denoted by $\mathcal{T}_{\ell}$, limited by the surfaces $\mathbf{S N}: A=G_{\ell}(\omega) \pm \lambda$ adjoining the graph of

$$
A=G_{\ell}(\omega), \quad \lambda=0
$$



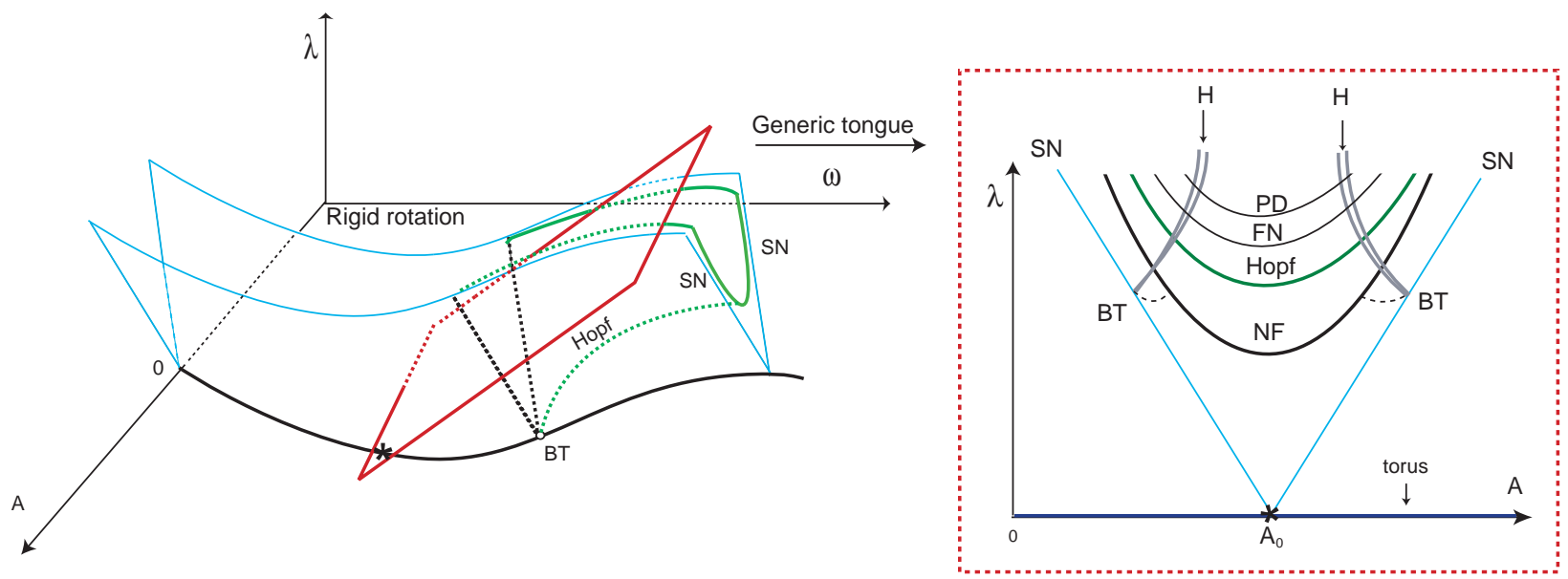

FigURE 13. Schematic representation of the bifurcations of fixed points of (5.4), giving rise to a resonance wedge (left) and an Arnold tongue (right) for $A>\lambda \gtrsim 0$. Bifurcations: BT: Bogdanov-Takens, SN: saddle-node, H: homoclinic tangencies, FN/NF: transitions focus $\leftrightarrow$ node, Hopf: Hopf, PD: perio d-doubling. This figure is distorted and nonlinearly scaled to enable some of the regions to be distinguished.

Parameters within this wedge correspond to first return maps with at least a pair of fixed points: one of the fixed points is a saddle (say $Q_{\ell}$ ); the other point is a sink (say $P_{\ell}$ ). As suggested in Figure 14, we suppose the existence of just one pair of fixed points for the following analysis, both with the same rotation number.

The borders of $\mathcal{T}_{\ell}$ are the bifurcation surfaces $\mathbf{S N}:=\mathbf{S N}_{\ell}^{1} \cup \mathbf{S N}_{\ell}^{2}$ at which the fixed points $Q_{\ell}$ and $P_{\ell}$ merge to a saddle-node. These surfaces might touch the corresponding surfaces of other wedge, meaning that there are parameter values for which periodic points of periods $m$ and $\ell$ coexist, $\ell, m \in \mathbb{N}$. The surface Hopf seems to connect both Hopf surfaces that appear near $\mathbf{B} \mathbf{T}_{\ell}^{1}$ and $\mathbf{B} \mathbf{T}_{\ell}^{2}$ given by Corollary $\mathrm{B}$, from where a stable 2-torus emerges. This torus is contractible because it does not envelope the cylinder $\overline{\operatorname{Out}\left(\mathrm{O}_{2}\right)}$; the new tori might coexist (for different values of $\ell \in \mathbb{N}$ ) and are not diffeomorphic to the original torus that exists for $\lambda=0$ and $A>0$.

In the bifurcation plane $(A, \lambda)$, the Hopf surface is above the set $\mathbf{N F}$ where the eigenvalues of the sink $P_{\ell}$ become complex. At the period-doubling bifurcation surface $\mathbf{P D}$, one multiplier becomes equal to -1 . At this stage, the closed curve resulting from the intersection of the torus with a global cross section, is no longer homeomorphic to a circle. Furthermore, the torus is no longer smooth as the unstable manifold of the saddle $Q_{\ell}$ winds around the focus infinitely many times (see Figure 14). Between the curves Hopf and PD, the eigenvalues of $P_{\ell}$ become real again, along a surface $\mathbf{F N}$. These bifurcations have been discussed in [43, 57] where the authors relate the dynamics of an Arnold tongue with maps on the circle.

Along the bifurcation surfaces $\mathbf{H}_{\ell}^{1}$ and $\mathbf{H}_{\ell}^{2}$ described by Corollary $\mathrm{B}$, one observes a homoclinic contact of the components $W^{s}\left(Q_{\ell}\right)$ and $W^{u}\left(Q_{\ell}\right)$, where $Q_{\ell}$ is a dissipative saddle for $\mathcal{F}_{\mu}$. There are small regions (in terms of measure) inside the resonance wedges where chaotic trajectories are observable: they correspond to strange attractors of Hénon type [52]. Other stable points of large period exist in the region above the surfaces $\mathbf{H}_{\ell}^{1}$ and $\mathbf{H}_{\ell}^{2}$, as a consequence of Newhouse phenomena [42]. Numerics in [17] also suggest the existence of 


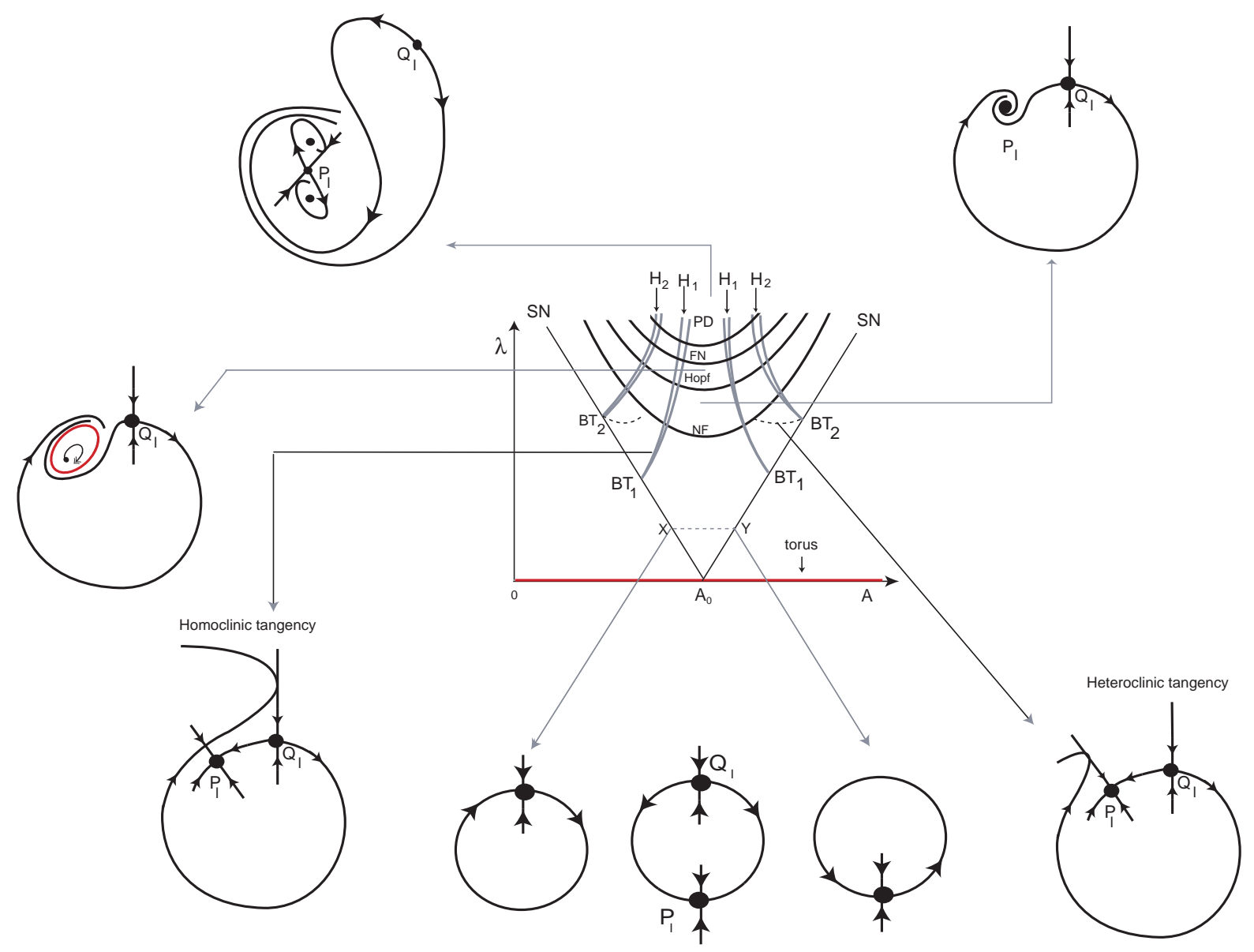

Figure 14. Plausible schematic representation of the Arnold tongue of Figure 13 emphasising the dynamics of $\mathcal{F}_{\mu}$. Bifurcations: $\mathbf{B T}_{1}, \mathbf{B T}_{2}$ : Bogdanov-Takens, SN: saddle-node, $\mathbf{H}_{1}, \mathbf{H}_{2}$ : homoclinic tangencies, $\mathbf{F N} / \mathbf{N F}$ : transitions focus $\leftrightarrow$ node, Hopf: Hopf, PD: period-doubling. The line defined by $\lambda=0$ corresponds to an attracting torus. Along the horizontal path $[X Y]$, trajectories change the way of rotation around the torus.

bistability for open regions of the parameter space: coexistence of a stable periodic solution and an attracting torus.

\section{An eXAmple}

Our study was initially motivated by the following example introduced in [4] and explored in [21. Some preliminaries about symmetries of a vector field may be found in Appendix A.1. For $\tau_{1}, \tau_{2} \in[0,1]$, our object of study is the two-parameter family of vector fields on $\mathbb{R}^{4}$

$$
x=\left(x_{1}, x_{2}, x_{3}, x_{4}\right) \in \mathbb{R}^{4} \mapsto f_{\left(\tau_{1}, \tau_{2}\right)}(x)
$$


defined for each $x=\left(x_{1}, x_{2}, x_{3}, x_{4}\right) \in \mathbb{R}^{4}$ by

$$
\left\{\begin{array}{l}
\dot{x}_{1}=x_{1}\left(1-r^{2}\right)-\omega x_{2}-\alpha x_{1} x_{4}+\beta x_{1} x_{4}^{2}+\tau_{2} x_{1} x_{3} x_{4} \\
\dot{x}_{2}=x_{2}\left(1-r^{2}\right)+\omega x_{1}-\alpha x_{2} x_{4}+\beta x_{2} x_{4}^{2} \\
\dot{x}_{3}=x_{3}\left(1-r^{2}\right)+\alpha x_{3} x_{4}+\beta x_{3} x_{4}^{2}+\tau_{1} x_{4}^{3}-\tau_{2} x_{1}^{2} x_{4} \\
\dot{x}_{4}=x_{4}\left(1-r^{2}\right)-\alpha\left(x_{3}^{2}-x_{1}^{2}-x_{2}^{2}\right)-\beta x_{4}\left(x_{1}^{2}+x_{2}^{2}+x_{3}^{2}\right)-\tau_{1} x_{3} x_{4}^{2}
\end{array}\right.
$$

where $\dot{x}_{i}=\frac{\partial x_{i}}{\partial t}, r^{2}=x_{1}^{2}+x_{2}^{2}+x_{3}^{2}+x_{4}^{2}$, and

$$
\omega>0, \quad \beta<0<\alpha, \quad \beta^{2}<8 \alpha^{2} \quad \text { and } \quad|\beta|<|\alpha| .
$$

The vector field $f_{(0,0)}$ is equivariant under the action of the compact Lie group $\mathbb{S O}(2)\left(\gamma_{\psi}\right) \oplus$ $\mathbb{Z}_{2}\left(\gamma_{2}\right)$, where $\mathbb{S O}(2)\left(\gamma_{\psi}\right)$ and $\mathbb{Z}_{2}\left(\gamma_{2}\right)$ act on $\mathbb{R}^{4}$ as

$$
\gamma_{\psi}\left(x_{1}, x_{2}, x_{3}, x_{4}\right)=\left(x_{1} \cos \psi-x_{2} \sin \psi, x_{1} \sin \psi+x_{2} \cos \psi, x_{3}, x_{4}\right), \quad \psi \in[0,2 \pi]
$$

given by a phase shift $\theta \mapsto \theta+\psi$ in the first two coordinates, and

$$
\gamma_{2}\left(x_{1}, x_{2}, x_{3}, x_{4}\right)=\left(x_{1}, x_{2},-x_{3}, x_{4}\right) .
$$

By construction, $\tau_{1}$ is the controlling parameter of the $\mathbb{Z}_{2}\left(\gamma_{2}\right)$-symmetry breaking and $\tau_{2}$ controls the $\mathbb{S O}(2)\left(\gamma_{\psi}\right)$-symmetry breaking but keeping the $\mathbb{S O}(2)\left(\gamma_{\pi}\right)$-symmetry, where

$$
\gamma_{\pi}\left(x_{1}, x_{2}, x_{3}, x_{4}\right)=\left(-x_{1},-x_{2}, x_{3}, x_{4}\right) .
$$

When restricted to the sphere $\mathbb{S}^{3}$, for every $\tau_{1}, \tau_{2} \in[0,1]$, the flow of $f_{\left(\tau_{1}, \tau_{2}\right)}$ has two equilibria

$$
O_{1}=(0,0,0,+1) \quad \text { and } \quad O_{2}=(0,0,0,-1),
$$

which are hyperbolic saddle-foci. The linearization of $f_{(0,0)}$ at $O_{1}$ and $O_{2}$ has eigenvalues

$$
-(\alpha-\beta) \pm \omega i, \alpha+\beta \quad \text { and } \quad(\alpha+\beta) \pm \omega i,-(\alpha-\beta)
$$

respectively. The $1 \mathrm{D}$-connections are given by:

$$
\begin{aligned}
\overline{W^{u}\left(O_{1}\right)} \cap \mathbb{S}^{3} & =\overline{W^{s}\left(O_{2}\right)} \cap \mathbb{S}^{3}=\operatorname{Fix}\left(\mathbb{S O O}(2)\left(\gamma_{\psi}\right)\right) \cap \mathbb{S}^{3} \\
& =\left\{\left(x_{1}, x_{2}, x_{3}, x_{4}\right): x_{1}=x_{2}=0, x_{3}^{2}+x_{4}^{2}=1\right\}
\end{aligned}
$$

and the 2D-connection is contained in

$$
\begin{aligned}
\overline{W^{u}\left(O_{2}\right)} \cap \mathbb{S}^{3} & =\overline{W^{s}\left(O_{1}\right)} \cap \mathbb{S}^{3}=\operatorname{Fix}\left(\mathbb{Z}_{2}\left(\gamma_{2}\right)\right) \cap \mathbb{S}^{3} \\
& =\left\{\left(x_{1}, x_{2}, x_{3}, x_{4}\right): x_{1}^{2}+x_{2}^{2}+x_{4}^{2}=1, x_{3}=0\right\} .
\end{aligned}
$$

The two-dimensional invariant manifolds of $O_{1}$ and $O_{2}$ are contained in the two-sphere $\operatorname{Fix}\left(\mathbb{Z}_{2}\left(\gamma_{2}\right)\right) \cap \mathbb{S}^{3}$. It is precisely the symmetry $\mathbb{Z}_{2}\left(\gamma_{2}\right)$ that forces the two-invariant manifolds $W^{u}\left(O_{2}\right)$ and $W^{s}\left(O_{1}\right)$ to coincide. We denote by $\Gamma$ the heteroclinic network formed by the two equilibria, the two connections $\left[O_{1} \rightarrow O_{2}\right]$ and the sphere $\left[O_{2} \rightarrow O_{1}\right]$. Keeping $\tau_{1}=\tau_{2}=0$, the equilibria $O_{1}$ and $O_{2}$ have the same chirality. Therefore:

Lemma 7.1. If $\tau_{1}=\tau_{2}=0$, the flow of (7.1) satisfies (P1)-(P5) described in Section 2.1.

As a consequence, for $\tau_{1}=\tau_{2}=0$, the flow of (7.1) exhibits an asymptotically stable heteroclinic network $\Gamma$ associated to $O_{1}$ and $O_{2}$. The parameters $\tau_{1}$ and $\tau_{2}$ play the role of $A$ and $\lambda$, respectively, of (P7) $-(\mathbf{P} 8)$, after possible rescaling.

Corollary 7.2. [21] For $\tau_{1}>0$ and $\tau_{2}=0$, close to the "ghost" of the attracting network $\Gamma$, the flow of (7.1) has an attracting invariant two-torus, which is normally hyperbolic. 
When $\tau_{1} \gg \tau_{2}>0$, although we break the $\mathbb{S O}(2)\left(\gamma_{\psi}\right)$-equivariance, the $\mathbb{Z}_{2}\left(\gamma_{\pi}\right)$-symmetry is preserved. This is why the connections lying in $x_{1}=x_{2}=0$ persist.

Lemma 7.3. 21] For $\tau_{1}, \tau_{2}>0$ small enough such that $\tau_{1} \gg \tau_{2}$, the flow of (7.1) satisfies (P7) $-(P 8 b)$.

Numerical simulations of (17.1) for $\tau_{1} \gg \tau_{2}>0$ suggest the existence of regular and chaotic behaviour in the region of transition from an attracting 2-dimensional torus to rotational horseshoes [21]. Chaotic attractors with one positive Lyapunov exponent seem to exist, as suggested by the yellow regions occurring in the upper part of the Arnold tongues in Figure 15. The description of Section 6 agrees quite well with the bifurcation diagram.

Hopf surfaces found in Corollary B correspond to the lower bound of the blue "bananas" that one observes in Figure 15. This bifurcation gives rise to a stable 2-torus (blue region) in the flow of (7.1). Numerically we lose control of this stable torus, although we guess that it persists in other location of the phase space. Our numeric findings show that there are tiny regions of the parameter space inside the resonance regions where strange attractors may be found.

Technicalities on numerics of Figure 15. The parameter plane $\left(\tau_{1}, \tau_{2}\right)$ of Figure [15] is scanned with a sufficiently small step along each coordinate axes. The software evaluates at each parameter value how many Lyapunov exponents along the orbit with initial condition $(0.1 ; 0.1 ; 0 ;-0.99) \in W^{u}\left(O_{2}\right)$, are non-negative (considered "positive" when greater than $5 \times 10^{-4}$ to discard uncertain positive Lyapunov exponents due to numerical precision issues). The parameter is painted according to the following rules: red for 0 , blue for 1 , yellow for 2. To estimate the complete Lyapunov spectra, the authors of [21] used the algorithm for differential equations with a Taylor series integrator.

\section{Discussion}

In this article, we have constructed a bifurcation diagram associated to a 3-parameter differential equation, whose starting point is a weakly attracting heteroclinic network with a 2-dimensional connecting manifold, a natural configuration in symmetric systems and in some unfoldings of the Hopf-zero singularity [12, 40, 54].

We concentrate our attention in a family of vector fields $f_{\mu} \in \mathfrak{X}^{3}\left(\mathbb{S}^{3}\right)$ satisfying (P7)(P8b)-(P9). The bifurcation diagram of each element of the family is governed by an Arnold wedge, a structure through which an Arnold tongue may be seen as a projection. This (new) heteroclinic bifurcation is different from that obtained in [7, in which an equilibrium produces a periodic solution which, in turn, generates a 2-torus.

The structure of the Arnold tongue strongly depends on $\omega$. This suggested us to extend the 2-parameter "classical" bifurcation diagram $\left(A, \frac{\lambda}{A}\right)$ of [52] to a 3-dimensional case, where $\omega$ is the additional parameter. Doing that, Arnold tongues give rise to resonance wedges bounded by two surfaces that correspond to saddle-node bifurcations. The resonance wedge contains a sequence of curves corresponding to a discrete-time Bogdanov-Takens bifurcation, a possibility already anticipated in [6, 31]. Parameters within the wedge corresponds to maps whose periodic orbits share the same rotation number.

The structure of a resonance wedge is consistent with the Torus-breakdown theory [1, 43, [46, 47], an essential route to understand the nature of turbulence [53]. When the speed of rotation $\omega>0$ is small, the flow of (2.1) exhibits an attracting torus consisting of either locked or quasiperiodic solutions. As $\omega$ increases, the attracting torus disintegrates into 


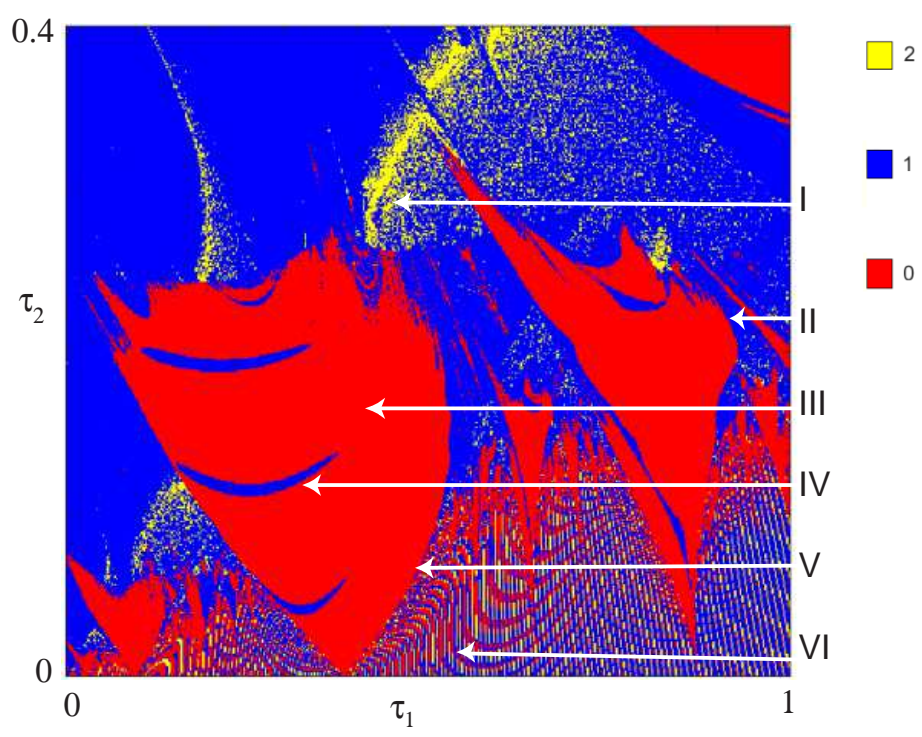

FIGURE 15. Number of non-negative Lyapunov exponents along the orbit with initial condition $(0.1 ; 0.1 ; 0 ;-0.99)$ near $W^{u}\left(O_{2}\right)$ for equation (7.1) with $\alpha=1, \beta=-0.1$ and $\omega=1, t \in[0,3750]$. Red for 0; Blue for 1; Yellow for 2. I - Homoclinic bifurcations; Hénon-like strange attractors; II - Sink; III - Resonant tongue (Arnold tongue); IV - Hopf bifurcation; V - Saddle-node bifurcation (border of the Arnold tongue); VI Irrational torus (thin yellow region). Figure performed by L. Castro adapted from 21].

isolated periodic sinks and saddles. Increasing the magnitude of $\omega$ further, the phase space is stretched and folded, creating rotational horseshoes, homoclinic tangencies and strange attractors of Hénon-type with ergodic SRB measures. In between, Hopf bifurcations are present. Our description refines the diagrams proposed by [9] and [10].

For $f_{\mu} \in \mathfrak{X}^{3}\left(\mathbb{S}^{3}\right)$, we may distinguish the dynamics between heteroclinic tangle and rankone like attractors; this depends on the hypotheses $(\mathbf{P} 8 \mathbf{a})$ and $(\mathbf{P} 8 \mathbf{b})$. In both cases, there exists complicated dynamics in $\mathcal{U}$, but chaotic dynamics are created by two independent mechanisms. They are:

$$
\begin{aligned}
\lambda>A \geq 0, \omega \in \mathbb{R}^{+} & \Leftrightarrow \quad \mathbf{P} \mathbf{8} \mathbf{a}): W^{u}\left(O_{2}\right) \text { and } W^{s}\left(O_{1}\right) \text { intersects transversely } \\
& \Rightarrow \text { the expansion induced by intersection of invariant manifolds } \\
& \Rightarrow \text { Smale horseshoes (heteroclinic tangles). } \\
A>\lambda \geq 0, \omega \in \mathbb{R}^{+} & \Leftrightarrow(\mathbf{P} \mathbf{b}): W^{u}\left(O_{2}\right) \text { and } W^{s}\left(O_{1}\right) \text { do not intersect } \\
& \Rightarrow \text { the invariant manifolds of the saddle-foci are pulled apart } \\
& \Rightarrow \text { the expansion is induced by large } \omega \\
& \Rightarrow \text { Rotational horseshoes. }
\end{aligned}
$$


In the first case, we conjecture the existence of a non-uniform expansion for a set with positive Lebesgue measure. The difficulties to prove the conjecture are linked with the existence of infinitely many points within $W^{s}\left(O_{1}\right)$ where the first return map is not well defined. In the second scenario, the dynamics is governed by strange attractors [56, 57]. A lot more needs to be done before these two types of chaos are well understood.

The analysis in this paper is not sensitive to the particular configuration given by the heteroclinic attractor $\Gamma$; the results are valid for more general weakly attracting networks with 2-dimensional heteroclinic connections which unfold generically from the coincidence. Finally, we would like to point out that all results also hold for periodically-forced differential equations, natural in the study of seasonally forced systems, where "our" parameters $A, \lambda, \omega$ may be interpreted as (cf. [39]):

A $\rightarrow$ Average of the periodic-forcing;

$\lambda \quad \rightarrow \quad$ Effect (fluctuations) of the unstable manifold on a global cross section;

$\omega \rightarrow$ Frequency of the forcing.

By moving parameters, the invariant manifolds of invariant saddles cause destruction and fusion of attractors. The fully description of these metamorphoses is under analysis and are deferred for future work.

\section{ACKNOWLEDGEMENTS}

The author is grateful to Isabel Labouriau for the fruitful discussions during the research work performed in [39]. Special thanks to Andrey Shilnikov for pointing out the paper [54] on bifurcations analysis of a low-order atmospheric circulation model. The author is indebted to the two reviewers for the constructive comments, corrections and suggestions which helped to improve the readability of this manuscript.

\section{REFERENCES}

[1] V.S. Afraimovich, L.P. Shilnikov. On invariant two-dimensional tori, their breakdown and stochasticity in: Methods of the Qualitative Theory of Differential Equations, Gor'kov. Gos. University (1983), 3-26. Translated in: Amer. Math. Soc. Transl., (2), vol. 149 (1991) 201-212.

[2] V.S. Afraimovich, S-B Hsu, H. E. Lin. Chaotic behavior of three competing species of May-Leonard model under small periodic perturbations. Int. J. Bif. Chaos, 11(2) (2001) 435-447.

[3] V. S. Afraimovich, S. B. Hsu. Lectures on Chaotic Dynamical Systems, American Mathematical Society and International Press, 2002.

[4] M. Aguiar. Vector fields with heteroclinic networks, Ph.D. thesis, Departamento de Matemática Aplicada, Faculdade de Ciências da Universidade do Porto, 2003.

[5] M.A.D. Aguiar, S.B.S.D. Castro, I.S. Labouriau. Dynamics near a heteroclinic network. Nonlinearity 18 (2005) 391-414.

[6] A. Algaba, M. Merino, A. Rodríguez-Luis. Takens-Bogdanov bifurcations of periodic orbits and Arnold's tongues in a three-dimensional electronic model, International Journal of Bifurcation and Chaos 11(02) (2001): 513-531.

[7] V. Anishchenko, M. Safonova, L Chua. Confirmation of the Afraimovich-Shilnikov torus-breakdown theorem via a torus circuit. IEEE Transactions on Circuits and Systems I: Fundamental Theory and Applications, 40(11) (1993) 792-800.

[8] V. Arnold. Small denominators. I. Mapping the circle onto itself, Izvestiya Rossiiskoi Akademii Nauk. Seriya Matematicheskaya 25(1) (1961) 21-86.

[9] D. Arrowsmith, J. Cartwright, A. Lansbury, C. Place. The Bogdanov map: Bifurcations, mode locking, and chaos in a dissipative system, International Journal of Bifurcation and Chaos 3.04 (1993): 803-842. 
[10] D. Aronson, M. Chory, G. Hall, R. McGehee. Bifurcations from an invariant circle for two-parameter families of maps of the plane: a computer-assisted study, Communications in Mathematical Physics, 83(3) (1982) 303-354.

[11] P. Ashwin, P. Chossat. Attractors for robust heteroclinic cycles with continua of connections, Journal of Nonlinear Science, 8(2) (1998) 103-129.

[12] I. Baldomá, S. Ibáñez, T. Seara. Hopf-Zero singularities truly unfold chaos, Commun. Nonlinear Sci. Numer. Simul. 84 (2020), 105162.

[13] T. Bakri, F. Verhulst. Bifurcations of quasi-periodic dynamics: torus breakdown. Zeitschrift für angewandte Mathematik und Physik 65.6 (2014) 1053-1076.

[14] T. Bakri, Y. Kuznetsov, F. Verhulst. Torus bifurcations in a mechanical system, Journal of Dynamics and Differential Equations, 27 (3-4), (2015) 371-403.

[15] P. L. Boyland. Bifurcations of circle maps: Arnold tongues, bistability and rotation intervals. Commun. Math. Phys., 106 (1986) 353-381.

[16] H. Broer, R. Roussarie, C. Simó. Invariant circles in the Bogdanov-Takens bifurcation for diffeomorphisms, Ergod. Th. \& Dynam. Sys. 16 (1996) 1147-1172.

[17] H. Broer, C. Simó, J. C. Tatjer. Towards global models near homoclinic tangencies of dissipative diffeomorphisms Nonlinearity 11 (1998) 667-770.

[18] V.V. Bykov. On systems with separatrix contour containing two saddle-foci. J. Math. Sci. 95 (1999) $2513-2522$.

[19] V.V. Bykov. Orbit Structure in a neighborhood of a separatrix cycle containing two saddle-foci. Amer. Math. Soc. Transl. 200 (2000) 87-97.

[20] M. J. Capiński, E. Fleurantin, J. M. James. Computer assisted proofs of two-dimensional attracting invariant tori for ODEs, Discrete \& Continuous Dynamical Systems-A, 2020 (to appear)

[21] M. L. Castro, A. A. P. Rodrigues. Torus-breakdown near a Bykov attractor: a case study, International Journal of Bifurcation and Chaos (2021), to appear.

[22] A. Denjoy. Sur les courbes définies par les équations différentielles a la surface du tore, J. Math. Pures Appl. 11 (1932) 333-375.

[23] P. Gaspard. Local birth of homoclinic chaos, Physica D: Nonlinear Phenomena, 62(1-4) (1993) 94-122.

[24] S.V. Gonchenko, L.P. Shilnikov, D.V. Turaev. Quasiattractors and homoclinic tangencies. Computers Math. Applic. 34(2-4) (1997) 195-227.

[25] B. Greenspan, P. Holmes. Repeated resonance and homoclinic bifurcation in a periodically forced family of oscillators, SIAM journal on mathematical analysis 15.1 (1984) 69-97.

[26] J. Guckenheimer, P. Holmes. Nonlinear Oscillations, Dynamical Systems, and Bifurcations of Vector Fields. Applied Mathematical Sciences 42, Springer-Verlag, 1983.

[27] M. Herman. Mesure de Lebesgue et Nombre de Rotation, Lecture Notes in Math., vol. 597, Springer, (1977) 271-293.

[28] M. Herman. Sur la conjugaison différentiable des difféomorphismes du cercle à des rotations, Publications Mathématiques de l'IHÉS 49 (1979) 5-233.

[29] M. W. Hirsch C. Pugh, M. Shub. Invariant manifolds. Bull. Amer. Math. Soc. 76, no. 5 (1970) $1015-1019$.

[30] A.J. Homburg, B. Sandstede. Homoclinic and Heteroclinic Bifurcations in Vector Fields. Handbook of Dynamical Systems 3, North Holland, Amsterdam (2010) 379-524.

[31] S. Kim, R. MacKay, J. Guckenheimer. Resonance regions for families of torus maps, Nonlinearity, 2(3) (1989) 391-404.

[32] V. Kirk. Merging of resonance tongues, Physica D: Nonlinear Phenomena 66. 3-4 (1993) $267-281$.

[33] V. Kirk, A. Rucklidge. The effect of symmetry breaking on the dynamics near a structurally stable heteroclinic cycle between equilibria and a periodic orbit, Dynamical Systems 23.1 (2008) 43-74.

[34] S. Kiriki, T. Soma. Takens' last problem and existence of non-trivial wandering domains, Advances in Mathematics, 306 (2017) 524-588.

[35] J. Knobloch, J.S.W. Lamb, K.N. Webster. Using Lin's method to solve Bykov's problems. J. Diff. Eqs. 257(8) (2014) 2984-3047.

[36] I.S. Labouriau, A.A.P. Rodrigues. Global generic dynamics close to symmetry. J. Diff. Eqs. 253(8) (2012) 2527-2557.

[37] I.S. Labouriau, A.A.P. Rodrigues. Dense heteroclinic tangencies near a Bykov cycle, J. Diff. Eqs. 259(12) (2015) 5875-5902. 
[38] I.S. Labouriau, A.A.P. Rodrigues. Global bifurcations close to symmetry. J. Math. Anal. Appl. 444(1) (2016) 648-671.

[39] I.S. Labouriau, A.A.P. Rodrigues. Bifurcations from an attracting heteroclinic cycle under periodic forcing, J. Diff. Eqs. 269(5) (2020) 4137-4174.

[40] W. F. Langford. Numerical studies of torus bifurcations. In: Numerical Methods for Bifurcation Problems. Birkhäuser, Basel, 1984. 285-295.

[41] L. Mora, M. Viana. Abundance of strange attractors, Acta Math. 171(1) (1993) 1-71.

[42] S.E. Newhouse. The abundance of wild hyperbolic sets and non-smooth stable sets for diffeomorphisms. Publ. Math. Inst. Hautes Études Sci. 50 (1979) 101-151.

[43] S. Ostlund, D. Rand, J. Sethna, E. Siggia. Universal properties of the transition from quasi-periodicity to chaos in dissipative systems. Physica D: Nonlinear Phenomena, 8(3), (1983) 303-342.

[44] I.M. Ovsyannikov, L.P. Shilnikov. On systems with a saddle-focus homoclinic curve. Math. USSR Sb. 58 (1987) 557-574.

[45] A. Passeggi, R. Potrie, M. Sambarino. Rotation intervals and entropy on attracting annular continua, Geometry \& Topology 22(4) (2018) 2145-2186.

[46] B. Peckham. The necessity of the Hopf bifurcation for periodically forced oscillators, Nonlinearity 3.2 (1990) 261-280.

[47] B. Peckham, C. Frouzakis, I. Kevrekidis. Bananas and banana splits: a parametric degeneracy in the Hopf bifurcation for maps. SIAM Journal on Mathematical Analysis, 26(1) (1995) 190-217.

[48] B. Peckham, I. Kevrekidis. Lighting Arnold flames: resonance in doubly forced periodic oscillators, Nonlinearity 15 (2002) 405-428.

[49] A.A.P. Rodrigues. Persistent switching near a heteroclinic model for the geodynamo problem. Chaos, Solitons \& Fractals 47 (2013) 73-86.

[50] A.A.P. Rodrigues. Repelling dynamics near a Bykov cycle. J. Dyn. Diff. Eqs. 25(3) (2013) 605-625.

[51] A. A. P. Rodrigues. Strange attractors and wandering domains near a homoclinic cycle to a bifocus, Journal of Differential Equations, 269(4) (2020), 3221-3258.

[52] A.A.P. Rodrigues. Unfolding a Bykov attractor: from an attracting torus to strange attractors, J. Dyn. Diff. Eqs. 2020 https://doi.org/10.1007/s10884-020-09858-z.

[53] D. Ruelle, F. Takens. On the nature of turbulence, Les rencontres physiciens-mathématiciens de Strasbourg-RCP25 12 (1971) 1-44.

[54] A. Shilnikov, G. Nicolis, C. Nicolis. Bifurcation and predictability analysis of a low-order atmospheric circulation model, Internat. J. Bifur. Chaos Appl. Sci. Engrg 5(06), (1995) 1701-1711.

[55] A. Shilnikov, L. P. Shilnikov, D. Turaev. On some mathematical topics in classical synchronization. A tutorial., Internat. J. Bifur. Chaos Appl. Sci. Engrg. 14 (2004) 2143-2160.

[56] Q Wang, A. Oksasoglu. Dynamics of homoclinic tangles in periodically perturbed second-order equations, J. Diff. Eqs. 250(2) (2011) 710-751.

[57] Q. Wang, L.S. Young. From Invariant Curves to Strange Attractors, Commun. Math. Phys. (2002) 225275.

[58] K. Yagasaki. Melnikov's method and codimension-two bifurcations in forced oscillations, J. Differential Equations, 185 (2002) 1-24.

\section{Appendix A. Glossary}

We record a miscellaneous collection of terms and terminology that are used throughout the text. For $\varepsilon>0$ small, consider the 3-parameter family of $C^{3}$-smooth autonomous differential equations

$$
\dot{x}=f_{(A, \lambda, \omega)}(x) \quad x \in \mathbb{S}^{3} \subset \mathbb{R}^{4} \quad A, \lambda \in[0, \varepsilon], \quad \omega \in \mathbb{R}^{+} .
$$

Since $\mathbb{S}^{3}$ is a compact set without boundary, the local solutions of (A.1) could be extended to $\mathbb{R}$. Denote by $\varphi_{(A, \lambda, \omega)}(t, x), t \in \mathbb{R}$, the associated flow.

A.1. Symmetry. Given a compact Lie group $\mathcal{G}$ of endomorphisms of $\mathbb{S}^{3}$, we will consider 3 -parameter families of vector fields $\left(f_{(A, \lambda, \omega)}\right)$ under the equivariance assumption

$$
f_{(A, \lambda, \omega)}(\gamma x)=\gamma f_{(A, \lambda, \omega)}(x)
$$


for all $x \in \mathbb{S}^{3}, \gamma \in \mathcal{G}$ and $(A, \lambda, \omega) \in[0, \varepsilon]^{2} \times \mathbb{R}^{+}$. For an isotropy subgroup $\widetilde{\mathcal{G}}<\mathcal{G}$, we write $\operatorname{Fix}(\widetilde{\mathcal{G}})$ for the vector subspace of points that are fixed by the elements of $\widetilde{\mathcal{G}}$. Note that, for $\mathcal{G}$-equivariant differential equations, the subspace $\operatorname{Fix}(\widetilde{\mathcal{G}})$ is flow-invariant.

A.2. Attracting set. A subset $\Omega$ of $\mathbb{S}^{3}$ for which there exists a neighborhood $U \subset \mathbb{S}^{3}$ satisfying $\varphi_{(A, \lambda, \omega)}(t, U) \subset U$ for all $t \geq 0$ and

$$
\bigcap_{t \in \mathbb{R}^{+}} \varphi_{(A, \lambda, \omega)}(t, U)=\Omega
$$

is called an attracting set by the flow. This set is not necessarily connected. Its basin of attraction, denoted by $\mathbf{B}(\Omega)$, is the set of points in $\mathbb{S}^{3}$ whose orbits have $\omega$-limit in $\Omega$. We say that $\Omega$ is asymptotically stable (or $\Omega$ is a global attractor) if $\mathbf{B}(\Omega)=\mathbb{S}^{3}$. An attracting set is said to be quasi-stochastic if it encloses periodic solutions with different Morse indices (dimension of the unstable manifold), structurally unstable cycles, sinks and saddle-type invariant sets (cf. [24]).

A.3. Heteroclinic structures. Suppose that $O_{1}$ and $O_{2}$ are two hyperbolic equilibria of (A.1) with different Morse indices (dimension of the unstable manifold). There is a heteroclinic cycle associated to $O_{1}$ and $O_{2}$ if

$$
W^{u}\left(O_{1}\right) \cap W^{s}\left(O_{2}\right) \neq \emptyset \quad \text { and } \quad W^{u}\left(O_{2}\right) \cap W^{s}\left(O_{1}\right) \neq \emptyset .
$$

For $i \neq j \in\{1,2\}$, the non-empty intersection of $W^{u}\left(O_{i}\right)$ with $W^{s}\left(O_{j}\right)$ is called a heteroclinic connection between $O_{i}$ and $O_{j}$, and will be denoted by $\left[O_{i} \rightarrow O_{j}\right]$. Although heteroclinic cycles involving equilibria are not a generic property within differential equations, they may be structurally stable within families of vector fields which are equivariant under the action of a compact Lie group $\mathcal{G} \subset \mathbb{O}(n)$, due to the existence of flow-invariant subspaces [26].

A heteroclinic cycle between two hyperbolic saddle-foci of different Morse indices, where one of the connections is transverse while the other is structurally unstable, is called a Bykov cycle. We address the reader to [30] for an overview of heteroclinic bifurcations and substantial information on the dynamics near different types of structures.

A.4. Historic behaviour. We say that the solution of (A.1), $\varphi_{(A, \lambda, \omega)}(t, x)$ with $x \in \mathbb{S}^{3}$, has historic behaviour if there is a continuous function $H: \mathbb{S}^{3} \rightarrow \mathbb{R}$ such that the time average $\frac{1}{T} \int_{0}^{T} H\left(\varphi_{(A, \lambda, \omega)}(t, x)\right) d t$ fails to converge.

A.5. Strange attractor. A (Hénon-type) strange attractor of a two-dimensional dissipative diffeomorphism $R$ defined in a Riemannian manifold $\mathcal{M}$, is a compact invariant set $\Lambda$ with the following properties:

- $\Lambda$ equals the closure of the unstable manifold of a hyperbolic periodic point;

- the basin of attraction of $\Lambda$ contains an open set;

- there is a dense orbit in $\Lambda$ with a positive Lyapounov exponent (exponential growth of the derivative along its orbit).

A vector field possesses a strange attractor if the first return map to a cross section does. 
A.6. SRB measure. Given an attracting set $\Omega$ for a continuous map $R: \mathcal{M} \rightarrow \mathcal{M}$ where $\mathcal{M}$ is a compact smooth manifold, consider the Birkhoff average with respect to the continuous function $T: \mathcal{M} \rightarrow \mathbb{R}$ on the $R$-orbit starting at $x \in \mathcal{M}$ :

$$
L(T, x)=\lim _{n \in \mathbb{N}} \frac{1}{n} \sum_{i=0}^{n-1} T \circ R^{i}(x) .
$$

Suppose that, for Lebesgue almost all points $x \in \mathbf{B}(\Omega)$, the limit (A.2) exists and is independent on $x$. Then $L$ is a continuous linear functional in the set of continuous maps from $\mathcal{M}$ to $\mathbb{R}$ (denoted by $C(\mathcal{M}, \mathbb{R})$ ). By the Riesz Representation Theorem, it defines a unique probability measure $\mu$ such that:

$$
\lim _{n \in \mathbb{N}} \frac{1}{n} \sum_{i=0}^{n-1} T \circ R^{i}(x)=\int_{\Omega} T d \mu
$$

for all $T \in C(\mathcal{M}, \mathbb{R})$ and for Lebesgue almost all points $x \in \mathbf{B}(\Omega)$. If there exists an ergodic measure $\mu$ supported in $\Omega$ such that $(\underline{A} .3)$ is satisfied for all continuous maps $T \in C(\mathcal{M}, \mathbb{R})$ for Lebesgue almost all points $x \in \mathbf{B}(\Omega)$, where $\mathbf{B}(\Omega)$ has positive Lebesgue measure, then $\mu$ is called a SRB (Sinai-Ruelle-Bowen) measure and $\Omega$ is a SRB attractor. More details in [57].

A.7. Non-trivial wandering domains. A non-trivial wandering domain for a given map $R$ on a Riemannian manifold $\mathcal{M}$ is a non-empty connected open set $D \subset \mathcal{M}$ which satisfies the following conditions:

- $R^{i}(D) \cap R^{j}(D)=\emptyset$ for every $i, j \geq 0(i \neq j)$

- the union of the $\omega$-limit sets of points in $D$ for $R$, denoted by $\Omega(D, R)$, is not equal to a single periodic orbit.

A wandering domain $D$ is called contracting if the diameter of $R^{n}(D)$ converges to zero as $n \rightarrow+\infty$.

A.8. Rotational horseshoe. Let $\mathcal{H}$ stand for the infinite annulus $\mathcal{H}=\mathbb{S}^{1} \times \mathbb{R}$ (endowed with the usual inner product from $\mathbb{R}^{2}$ ). We denote by $\mathrm{Homeo}^{+}(\mathcal{H})$ the set of homeomorphisms of the annulus which preserve orientation. Given a homeomorphism $f: X \rightarrow X$ and a partition of $m \in \mathbb{N} \backslash\{1\}$ elements $R_{0}, \ldots, R_{m-1}$ of $X \subset \mathcal{H}$, the itinerary function $\xi: X \rightarrow$ $\{0, \ldots, m-1\}^{\mathbb{Z}}=\Sigma_{m}$ is defined by:

$$
\xi(x)(j)=k \quad \Leftrightarrow \quad f^{j}(x) \in R_{k}, \quad \text { for every } \quad j \in \mathbb{Z} .
$$

Following [45], we say that a compact invariant set $\Lambda \subset \mathcal{H}$ of $f \in \mathrm{Homeo}^{+}(\mathcal{H})$ is a rotational horseshoe if it admits a finite partition $P=\left\{R_{0}, \ldots, R_{m-1}\right\}$ by sets $R_{i}$ with non empty interior in $\Lambda$ so that:

- the itinerary $\xi$ defines a semi-conjugacy between $\left.f\right|_{\Lambda}$ and the full-shift $\sigma: \Sigma_{m} \rightarrow \Sigma_{m}$, that is $\xi \circ f=\sigma \circ \xi$ with $\xi$ continuous and onto;

- for any lift $F: \mathbb{R}^{2} \rightarrow \mathbb{R}^{2}$ of $f$, there exist $k>0$ and $m$ vectors $v_{0}, \ldots, v_{m-1} \in \mathbb{Z} \times\{0\}$ so that:

$$
\left\|\left(F^{n}(\hat{x})-\hat{x}\right)-\sum_{i=0}^{n} v_{\xi(x)(i)}\right\|<k \quad \text { for every } \quad \hat{x} \in \pi^{-1}(\Lambda), \quad n \in \mathbb{N},
$$


where $\|\star\|$ is the usual norm on $\mathbb{R}^{2}, \pi: \mathbb{R}^{2} \rightarrow \mathcal{H}$ denotes the usual projection map and $\hat{x} \in \pi^{-1}(\Lambda)$ is the lift of $x$; more details in the proof of Lemma 3.1 of [45]. The existence of a rotational horseshoe for a map implies positive topological entropy at least $\log m$.

Alexandre Rodrigues, Centro de Matemática da Univ. do Porto, Rua do Campo Alegre, 687, 4169-007 Porto, Portugal

Email address: alexandre.rodrigues@fc.up.pt 